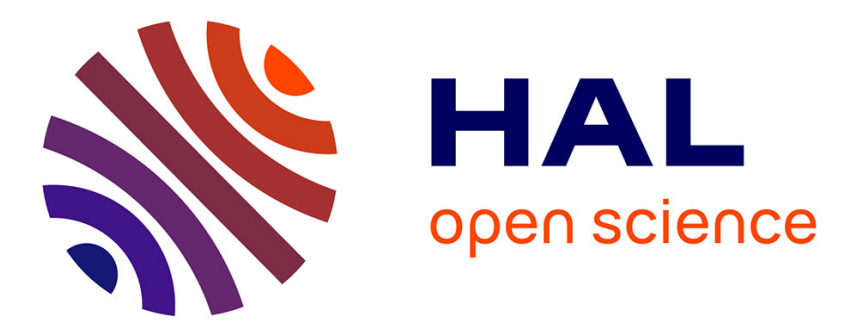

\title{
A stochastic target formulation for optimal switching problems in finite horizon
}

Bruno Bouchard

\section{To cite this version:}

Bruno Bouchard. A stochastic target formulation for optimal switching problems in finite horizon.

Stochastics and Stochastics Reports, 2009, 81 (2), pp.171-197. hal-00090377

\section{HAL Id: hal-00090377 \\ https://hal.science/hal-00090377}

Submitted on 30 Aug 2006

HAL is a multi-disciplinary open access archive for the deposit and dissemination of scientific research documents, whether they are published or not. The documents may come from teaching and research institutions in France or abroad, or from public or private research centers.
L'archive ouverte pluridisciplinaire HAL, est destinée au dépôt et à la diffusion de documents scientifiques de niveau recherche, publiés ou non, émanant des établissements d'enseignement et de recherche français ou étrangers, des laboratoires publics ou privés. 


\title{
A stochastic target formulation for optimal switching problems in finite horizon
}

\author{
Bruno Bouchard* \\ Université Paris VI, LPMA, and CREST, Paris, France
}

July 2006

\begin{abstract}
We consider a general optimal switching problem for a controlled diffusion and show that its value coincides with the value of a well suited stochastic target problem associated to a diffusion with jumps. The proof consists in showing that the Hamilton-Jacobi-Bellman equations of both problems are the same and in proving a comparison principle for this equation. This provides a new family of lower bounds for the optimal switching problem which can be computed by Monte-Carlo methods. This result has also a nice economical interpretation in terms of firm's valuation.
\end{abstract}

Keywords : Optimal switching, impulse control, stochastic targets, jump diffusion processes, viscosity solutions, comparison principle.

MSC Classification (2000) : 62L15, 49L25, 49L20.

\section{Introduction}

In this paper, we consider two very different stochastic control problems.

1. First, we discuss a general optimal switching problem in finite horizon. The aim is to maximize the expectation of a gain depending on the terminal value and the path of a Brownian diffusion process $X$ whose coefficients may have different regimes that are switched at stopping times decisions. This gain also incorporates a "cost" (positive or negative) which is due to the different switches.

Such formulations have been widely used in the economic literature to model firm's investment and management problems under uncertainty when a finite number of operating modes can be chosen, see e.g. [4], [8], [10] and [9]. From the mathematical point of view, they lead to the formulation of variational PDEs, see e.g. [1], [13], [19] and the above references, which is well understood:

*bouchard@ccr.jussieu.fr, http://www.proba.jussieu.fr/pageperso/bouchard/ 
(a) Let $v$ denote the associated value function. If a switch is performed while $X$ was evolving according to the mode $e$, then, by optimality, the value of $v$ before the switch, say $v(\cdot, e)$, must be at least equal to its value after the switch, say to $j$, diminished by the induced "cost", say $c_{e j}: v(\cdot, e) \geq v(\cdot, j)-c_{e j}$. Intuitively, equality holds if and only if it is optimal to switch from $e$ to $j$.

(b) If no switch is performed, then no cost is paid and the coefficients of the diffusion do not change. The value function $v$ then just follows the usual equation $\mathcal{L}^{X, e} v=0$ where $\mathcal{L}^{X, e}$ denotes the Dynkin operator associated to $X$ in the mode $e$.

2. Second, we study a particular class of stochastic target problems associated to a mixed diffusion process. Stochastic target problems have attracted a lot of attention in the financial literature because they are related to the super-replication of contingent claims, see e.g. the discussions and references in [2], or [11] for a more financial presentation.

The general formalism is the following: given a process $Z^{z, \nu}$ with initial condition $z$ and depending on some control $\nu$, find the set $\Gamma$ of initial conditions $z$ such that there exists an admissible $\nu$ for which $Z_{T}^{z, \nu}$ belongs a.s. to a prescribed fixed Borel set, called the target.

Recently, [16] proposed a direct dynamic programming principle which allows to solve such problems by PDE techniques in Brownian diffusion models, see [17]. The case of jump diffusion models was then considered in [2].

Let us discuss the particular case where $Z^{z, \nu}$ is of the form $\left(Y^{(y, x, e), \nu}, X^{(x, e)}, N^{e}\right)$ with values in $\mathbb{R} \times \mathbb{R}^{d} \times E$, where $N^{e}$ is a pure jump process and $E$ is a finite set. We fix $(x, e)$ and only write $\left(Y^{y, \nu}, X, N\right)$. If the target is the epigraph of some Borel map $g$ and the size of the jump of $Y$ does not depend on $Y$, then $\Gamma$ is a half-space and we can introduce the value function $u(0, x, e)$ defined as the minimal value of $y$ for which there is a control $\nu$ satisfying $Y_{T}^{y, \nu} \geq g\left(X_{T}, N_{T}\right)$. Letting $u(t, x, e)$ be defined similarly if we start from $t$, then the intuition says that we should always have $Y_{t}^{y, \nu} \geq u\left(t, X_{t}, N_{t}\right)$ (this is actually a part of the direct dynamic programming principle of [17]). This allows to draw the following conclusion: $d Y_{0}^{y, \nu} \geq d u\left(0, X_{0}, N_{0}\right)$ if $y=u(0, x, e)$. Let us assume that $\left(Y^{y, \nu}, X\right)$ has a jump $\left(c_{e, j}, 0\right)$ if and only if $N$ jumps from $e$ to $j$. Then:

(a') If $N$ jumps to $j$ at $0+$, we must have $c_{e, j} \geq u(0, x, j)-u(0, x, e)$.

(b') If $N$ does not jump at $0+$, we must have $d Y_{0}^{y, \nu}=d u\left(0, X_{0}, e\right)$, at least at the level of the Brownian components. Assuming that we have equality by optimality of $y$ and that the drift of $Y^{y, \nu}$ is zero, this implies that $\mathcal{L}^{X, e} u(0, x, e)=0$ where $\mathcal{L}^{X, e}$ is the Dynkin operator associated to $X$ when $N=e$.

Comparing (a)-(b) to (a')-(b') shows that we formally end up with the same class of variational PDE's. Otherwise stated, the value functions should coincide when the coefficients are well chosen. 
In this paper, we give a sens to this assertion. We first provide a suitable PDE characterization for the value function $v$ of a fairly general optimal switching problem. To the best of our knowledge, this derivation is new, and of own interest. We then introduce a well-suited stochastic target problem whose value function $u$ is bounded from below by the value $\tilde{u}$ of a natural dual problem. We show that $u$ (resp. $\tilde{u}$ ) is a subsolution (resp. supersolution) of the PDE associated to $v$. We then prove a comparison principle which allows us to conclude that $v=u=\tilde{u}$, i.e. the value functions coincide.

This provides two alternative formulations for the optimal switching problem. The first one has a nice economic interpretation in terms of firm's valuation, see below. The second means that the sup over switching controls can be replaced by a sup over a parameterized family of probability measures which leave unchanged the underlying Brownian motion. This provides a new family of lower bounds which can be computed by Monte-Carlo methods.

To conclude this introduction, we would like to observe that our representation of variational PDEs by stochastic target problems is in the spirit of [18] where systems of quasi-linear PDEs are related to Backward SDEs with jumps, see also [14] and [3] for numerical applications. The difference with [18] is that, in our setting, the jump part of the $Y$ process is not controlled, see below.

The rest of the paper is organized as follows. The two problems and our main result are exposed in Section 2. The PDE characterization of the value functions is given in Section 3. The technical proofs are collected in Section 4. The last section contains examples of conditions on the coefficients under which our general assumptions are satisfied.

In the following, all inequalities involving random variables have to be taken in the $\mathbb{P}$-a.s. sens. The quantity $|x|$ denotes the Euclidian norm of $x \in \mathbb{R}^{d}, B(x, r)$ is the open ball centered in $x$ with radius $r>0, \partial B(x, r)$ its boundary and $y^{+}$(resp. $y^{-}$) denotes the positive (resp. negative) part of $y \in \mathbb{R}$. .

\section{Problems formulation and main results}

Let $(\Omega, \mathcal{F}, \mathbb{P})$ be a complete probability space endowed with a filtration $\mathbb{F}=\left(\mathcal{F}_{t}\right)_{t \leq T}$ which is assumed to satisfy the usual conditions. Here, $T>0$ is a fixed time horizon.

\subsection{The optimal switching problem}

Given a finite set $E=\{0, \ldots, \kappa\}$ and $e_{0} \in E$, we say that a $\mathbb{F}$-adapted process $\xi$ is a $E$-valued switching control with initial condition $e_{0} \in E$ if it is of the form

$$
\xi_{t}=e_{0}+\sum_{i \geq 1} \mathcal{E}_{i} \mathbf{1}_{\tau_{i} \leq t<\tau_{i+1}} \quad, t \leq T,
$$


where $\left(\tau_{i}\right)_{i \geq 1}$ is an increasing sequence of stopping times satisfying $\tau_{i} \rightarrow \infty \mathbb{P}-$ a.s., and $\left(\mathcal{E}_{i}\right)_{i \geq 1}$ is a sequence of $E$-valued random variables such that $\mathcal{E}_{i}$ is $\mathcal{F}_{\tau_{i}}$-measurable for each $i \geq 1$. For such a process $\xi$, we denote by $\left(\tau_{i}^{\xi}\right)_{i \geq 1}$ the associated sequence of stopping times.

Let $W$ be a $\mathbb{R}^{d}$-valued standard Brownian motion. Given $X_{0} \in \mathbb{R}^{d}$, we consider the controlled process $X^{\xi}$ defined as the solution of the stochastic differential equation

$$
X_{t}=X_{0}+\int_{0}^{t} b\left(X_{s}, \xi_{s}\right) d s+\int_{0}^{t} a\left(X_{s}, \xi_{s}\right) d W_{s}+\sum_{\tau_{i}^{\xi} \leq t} \beta\left(X_{\tau_{i}^{\xi-}}, \xi_{\tau_{i}^{\xi}-}, \xi_{\tau_{i}^{\xi}}\right)
$$

Here, $b, a$ (resp. $\beta$ ) are assumed to be uniformly Lipschitz continuous on $\mathbb{R}^{d} \times[0, \kappa]$ (resp. $\left.\mathbb{R}^{d} \times[0, \kappa]^{2}\right)$ so that $X^{\xi}$ is well defined. We also assume that there is some $\Psi$ defined on $E^{2}$ such that

$$
\sup _{x \in \mathbb{R}^{d}}|\beta(x, i, j)| \vee 1 \leq \Psi(i, j) \quad \text { for all } \quad i, j \in E,
$$

and we say that a $E$-valued switching control $\xi$ is admissible if

$$
\mathbb{E}\left[\left|\sum_{\tau_{i}^{\xi} \leq T} \Psi\left(\xi_{\tau_{i}^{\xi}-}, \xi_{\tau_{i}^{\xi}}\right)\right|^{2 \bar{p}}\right]<\infty
$$

where $\bar{p} \geq 1$ is a fix parameter. We denote by $\mathcal{S}_{0}\left(e_{0}\right)$ the set of admissible $E$-valued switching control satisfying $\xi_{0}=e_{0}$. Using standard arguments based on BurkholderDavis-Gundy's inequality, Gronwall's Lemma and (2.3), one easily checks that

$$
\mathbb{E}\left[\sup _{t \leq T}\left|X_{t}^{\xi}\right|^{2 \bar{p}}\right]<\infty \quad \text { for all } \xi \in \mathcal{S}_{0}\left(e_{0}\right)
$$

This ensures that the negative part of the quantity

$$
\Pi(\xi):=g\left(X_{T}^{\xi}, \xi_{T}\right)+\int_{0}^{T} f\left(X_{s}^{\xi}, \xi_{s}\right) d s-\sum_{\tau_{i}^{\xi} \leq T} c\left(X_{\tau_{i}^{\xi}-}^{\xi}, \xi_{\tau_{i}^{\xi}-}, \xi_{\tau_{i}^{\xi}}\right)
$$

is integrable under the standing assumption

$$
\sup _{(x, i, j) \in \mathbb{R}^{d} \times E^{2}} \frac{|g(x, i)|+|f(x, i)|+|c(x, i, j)|}{1+|x|^{\bar{p}}}<\infty
$$

and

$$
\sup _{x \in \mathbb{R}^{d}} c(x, i, j)^{+} \leq \Psi(i, j) \quad \text { for all } \quad i, j \in E
$$


We can then introduce the optimal switching problem

$$
v\left(0, X_{0}, e_{0}\right):=\sup _{\xi \in \mathcal{S}_{0}\left(e_{0}\right)} \mathbb{E}[\Pi(\xi)] .
$$

In all this paper, we shall also assume that $g, f$ and $c$ are locally Lispchitz and that

$$
\beta(\cdot, e, e)=0 \quad \text { and } \quad c(\cdot, e, e)=1 \quad \text { for all } e \in E .
$$

This condition is natural and can be seen as a convention. It prevents from "optimal" strategies $\xi$ for which $\mathbb{P}\left[\exists i \geq 1\right.$ s.t. $\tau_{i}^{\xi} \leq T$ and $\left.\xi_{\tau_{i}^{\xi}-}=\xi_{\tau_{i}^{\xi}}\right]>0$. We could alternatively impose that admissible controls satisfy $\xi_{\tau_{i}^{\xi}} \neq \xi_{\tau_{i}^{\xi}} \mathbb{P}-$ a.s. for each $i \geq 1$.

Remark 2.1 As in [19], we work with a finite time horizon and the instantaneous reward function may depend on the current regime. The novelty of this paper is that:

(i) the switching process may have an impact on the terminal reward function and the size of the jumps of the diffusion,

(ii) the jump coefficients may depend on the current value of the diffusion process,

(iii) as in [13], which corresponds to an infinite horizon problem, the cost function $c$ is not assumed to be positive (nor non-negative).

We also make less restrictive assumptions on the coefficients. However, we should point out that our model does not include other types of control as in [19], and is therefore less general from this point of view.

Remark 2.2 Let $\psi: \mathbb{R}^{d} \times E \mapsto \psi(x, e)$ be Lipschitz in its first variable uniformly. Assume further that, for some $C>0$,

$$
|\psi(\cdot, i)-\psi(\cdot, j)| \leq C \quad \text { for all } i, j \in E .
$$

Then, $\psi=\tilde{\psi}$ on $\mathbb{R}^{d} \times E$ where $\tilde{\psi}$ is defined on $\mathbb{R}^{d} \times[0, \kappa]$ by

$$
\tilde{\psi}(x, y)=\psi(x, \kappa) \mathbf{1}_{y=\kappa}+\sum_{i=0}^{\kappa-1}\{\psi(x, i)(i+1-y)+\psi(x, i+1)(y-i)\} \mathbf{1}_{i \leq y<i+1}
$$

and is uniformly Lipschitz on $\mathbb{R}^{d} \times[0, \kappa]$. Hence, we could alternatively assume that $b, a, \beta$ are uniformly Lipschitz in their first variable and satisfy condition (2.10). The same remark holds for the local Lipschitz assumption on $g, f$ and $c$.

\subsection{The stochastic target problem}

\subsubsection{Primal formulation}

The alternative formulation for (2.8) is constructed as follows. First, we introduce a random measure $\mu$ associated to a $E$-valued point process. We assume that it admits a 
predictable $(\mathbb{P}, \mathbb{F})$-intensity of the form $\sum_{k=0}^{\kappa} \delta_{k}(\sigma) d \sigma d t$ where $\delta_{k}$ denotes the Dirac mass at $k$, and that it is independent of $W$, see e.g. [5]. We then define the $\mathbb{F}$-adapted process $N$ by

$$
N_{t}=\left[e_{0}+\int_{0}^{t} \int_{E} \sigma \mu(d \sigma, d s)\right]_{\kappa}, t \leq T
$$

where $[\cdot]_{\kappa}$ means that the term in brackets is taken modulo $\kappa+1$. Observe that $N \in$ $\mathcal{S}_{0}\left(e_{0}\right)$.

We next introduce the set $\Phi$ of $\mathbb{R}^{d}$-valued $\mathbb{F}$-predictable processes $\phi$ such that

$$
\mathbb{E}\left[\int_{0}^{T}\left|\phi_{s}\right|^{2} d s\right]<\infty
$$

and, given $(y, \phi) \in \mathbb{R} \times \Phi$, we set

$$
Y_{t}^{y, \phi}=y-\int_{0}^{t} f\left(X_{s}^{N}, N_{s}\right) d s+\int_{0}^{t} \phi_{s} \cdot d W_{s}+\sum_{\tau_{i}^{N} \leq t} c\left(X_{\tau_{i}^{N}-}^{N}, N_{\tau_{i}^{N}-}, N_{\tau_{i}^{N}}\right),
$$

where $\cdot$ stands for the scalar product. The stochastic target problem is:

$$
u\left(0, X_{0}, e_{0}\right):=\inf \left\{y \in \mathbb{R}: \exists \phi \in \Phi \text { s.t. } Y_{T}^{y, \phi} \geq g\left(X_{T}^{N}, N_{T}\right)\right\} .
$$

Remark 2.3 This problem almost fits in the general framework of [2]. Up to now the only difference is that our control process $\phi$ does not evolve in a compact set (but the fact that it appears only in the martingale part of $Y$ actually makes the proofs easier). An other difference will appear below in the definition of viscosity solutions, see Definition 3.1 , which is slightly different from the one used in [2].

It follows from standard arguments that

$$
\mathbb{E}\left[\sup _{t \leq T}\left|X_{t}^{N}\right|^{p}+\Pi(N)^{p}\right]<\infty \quad \text { for all } \quad p \geq 1 .
$$

Moreover, it is clear that $u\left(0, X_{0}, e_{0}\right)$ coincides with the minimal value of $y$ for which there exists some $\phi \in \Phi$ satisfying

$$
y+\int_{0}^{T} \phi_{s} \cdot d W_{s} \geq \Pi(N) .
$$

\subsubsection{Dual formulation}

We conclude this section, with the introduction of a suitable dual problem associated to $u$. Let $\mathcal{U}$ denote the set of predictable essentially bounded processes $\nu=\left(\nu^{0}, \ldots, \nu^{\kappa}\right)$ with values in $(0, \infty)^{\kappa+1}$. Given $\nu \in \mathcal{U}$, we set

$$
H_{0}^{\nu}:=\mathcal{E}\left(\int_{0}^{T} \int_{E}\left(\nu_{t}^{\sigma}-1\right) \tilde{\mu}(d \sigma, d t)\right)
$$


where $\mathcal{E}$ denotes the Doléans-Dade exponential and $\tilde{\mu}$ the compensated measure associated to $\mu$. We denote by $\mathbb{Q}^{\nu}$ the equivalent probability measure satisfying $d \mathbb{Q}^{\nu} / d \mathbb{P}=H_{0}^{\nu}$, see e.g. [5].

Observing that each $H_{0}^{\nu}, \nu \in \mathcal{U}$, can be approximated in $L^{2}(\Omega, \mathbb{P})$ by a sequence of $\left(H_{0}^{\nu_{n}}\right)_{n \geq 1}, \nu_{n} \in \mathcal{U}$, of essentially bounded densities, we deduce from (2.14) and (2.15) that

$$
u\left(0, X_{0}, e_{0}\right) \geq \tilde{u}\left(0, X_{0}, e_{0}\right):=\sup _{\nu \in \mathcal{U}} \mathbb{E}^{\mathbb{Q}^{\nu}}[\Pi(N)]
$$

where $\mathbb{E}^{\mathbb{Q}^{\nu}}$ is the expectation operator under $\mathbb{Q}^{\nu}$. In the following, we shall prove that equality holds in (2.16). This result is standard in mathematical finance and is in the spirit of [7], [11] and [12]. However the conditions of these papers are not satisfied here, in particular because $\Pi(N)$ is not bounded from below and the set of admissible strategies we consider is different.

\subsection{The equivalence result}

The main result of this paper relates $v, u$ and $\tilde{u}$ and thus provides a relation between optimal switching problems and a class of stochastic target problems. The alternative characterization of $v$ is obtained under the additional assumptions $\mathbf{H 1}$ and $\mathbf{H 2}$ whose precise definitions will be given in the next section.

Theorem 2.1 Assume that the conditions $\mathbf{H 1}$ and $\mathbf{H 2}$ of Section 3.3 are in force. Then, the three value functions coincide, i.e. $v\left(0, X_{0}, e_{0}\right)=u\left(0, X_{0}, e_{0}\right)=\tilde{u}\left(0, X_{0}, e_{0}\right)$.

Proof. The equality of the three value functions is a consequence of the PDE characterization of $v, u$ and $\tilde{u}$ reported in Theorem 3.1 below.

Remark 2.4 (Mathematical interpretation) The mathematical interpretation of this representation is the following. By changing $\mathbb{Q}^{\nu}, \nu \in \mathcal{U}$, we control the intensity and the law of the size of the jumps of $N$. This is formally equivalent to choosing a new element $\tilde{N}$ in $\mathcal{S}_{0}\left(e_{0}\right)$ and computing the expectation in (2.8) for $\xi=\tilde{N}$. This implies that $v\left(0, X_{0}, e_{0}\right)$ is bounded from below by $\tilde{u}\left(0, X_{0}, e_{0}\right)$. On the other hand, we can consider a sequence $\nu^{n}$ in $\mathcal{U}$ such that the predictable intensity of $N$ under $\mathbb{Q}^{\nu^{n}}$ tends to $\sum_{\tau_{i}} \delta_{\xi_{\tau_{i}}}(\sigma) \delta_{\tau_{i}^{\xi}}(t) d \sigma d t$ for any $\xi \in \mathcal{S}_{0}\left(e_{0}\right)$, see the proof of Proposition 4.7 below. This formally implies that $\tilde{u}\left(0, X_{0}, e_{0}\right)$ is also an upper-bound for $v\left(0, X_{0}, e_{0}\right)$.

Remark 2.5 (Numerical implication) Observe that the representation $v=\tilde{u}$ provides a natural and simple numerical method to construct a lower bound for $v$. Once a measure $\mathbb{Q}^{\nu}, \nu \in \mathcal{U}$, is chosen, we can approximate $\left(X^{N}, N\right)$ by a standard Euler scheme and estimate the expectation in (2.16) by its Monte-Carlo counterpart. Clearly, 
the discussion of the previous remark shows that we can not expect to obtain existence in the right hand-side of (2.16) so that the Monte-Carlo estimator can only give a lower bound. However, we may hope to provide a sharp estimate, for instance by considering a parametric family in $\mathcal{U}$ and using Robbins-Monro type optimization algorithms.

Remark 2.6 (Economic interpretation) The formulation $v=u$ has a nice economic interpretation. Optimal switching problems are widely used to model industrial issues such as the optimal management of power plants, see the references given in the introduction. The quantity $\Pi(\xi)$ represents the net production gain associated to a management rule $\xi \in \mathcal{S}_{0}\left(e_{0}\right)$ and $v\left(0, X_{0}, e_{0}\right)$ is often interpreted as the value of the firm. The equality $v=u$ actually gives a sens to this assertion.

Let us consider a Black-Scholes financial market with zero interest rate and $d$ risky assets $S=\left(S^{1}, \ldots, S^{d}\right)$ whose dynamics is given by

$$
S_{t}:=S_{0}+\int_{0}^{t} \operatorname{diag}\left[S_{s}\right] \Sigma d W_{s}
$$

where $\operatorname{diag}[x]$ is the diagonal matrix whose $i$-th diagonal element is $x^{i}$. A financial strategy is an element of $\Phi$ which represents the amount invested in each asset. The wealth process $Z^{y, \phi}$ associated to $\phi$ and initial wealth $y$ is given by

$$
Z_{t}^{y, \phi}=y+\int_{0}^{t} \phi_{s} \cdot \operatorname{diag}\left[S_{s}\right]^{-1} d S_{s}=y+\int_{0}^{t} \phi_{s} \cdot \Sigma d W_{s} .
$$

If $\Sigma$ is assumed to be invertible, then it is clear that $u\left(0, X_{0}, e_{0}\right)$ is the minimal value of $y$ such that $Z_{T}^{y, \phi} \geq \Pi(N)$ for some $\phi \in \Phi$. Otherwise stated, it follows from Theorem 2.1 , that $v\left(0, X_{0}, e_{0}\right)$ coincides with the super-hedging price of the net production gain associated to a random management rule $N$, which may correspond to the point of view of a financial agent who can not control how the company will actually be managed. We refer to [11] for an introduction to the notion of super-hedging in finance.

\section{Viscosity characterization of the value functions}

\subsection{Problem extension}

As usual, we extend the definition of the value functions to general initial conditions. We fix $(t, x) \in[0, T] \times \mathbb{R}^{d}$.

1. Given $\xi \in \mathcal{S}_{0}:=\cup_{e \in E} \mathcal{S}_{0}(e)$, let $X^{(t, x), \xi}$ denote the solution of (2.1) on $[t, T]$ satisfying $X_{t}^{(t, x), \xi}=x$ and set

$$
v(t, x, e):=\sup _{\xi \in \mathcal{S}_{t}(e)} \mathbb{E}\left[\Pi^{t, x}(\xi)\right]
$$


where $\mathcal{S}_{t}(e)=\left\{\xi \in \mathcal{S}_{0}: \xi_{t}=e\right\}$ and

$$
\Pi^{t, x}(\xi):=g\left(X_{T}^{(t, x), \xi}, \xi_{T}\right)+\int_{t}^{T} f\left(X_{s}^{(t, x), \xi}, \xi_{s}\right) d s-\sum_{t<\tau_{i}^{\xi} \leq T} c\left(X_{\tau_{i}^{\xi}-}^{(t, x), \xi}, \xi_{\tau_{i}^{\xi}-}, \xi_{\tau_{i}^{\xi}}\right) .
$$

2. For $e \in E$, we also define

$$
N_{s}^{t, e}=\left[e+\int_{t}^{s} \int_{E} \sigma \mu(d \sigma, d r)\right]_{\kappa}
$$

and we write $X^{t, x, e}$ for $X^{(t, x), N^{t, e}}, \tau_{i}^{t, e}$ for $\tau_{i}^{N^{t, e}}$. Given $(y, \phi) \in \mathbb{R} \times \Phi$, we next define $Y^{(t, x, e, y), \phi}$ on $[t, T]$ by

$Y_{s}^{(t, x, e, y), \phi}=y-\int_{t}^{s} f\left(X_{r}^{t, x, e}, N_{r}^{t, e}\right) d r+\int_{t}^{s} \phi_{r} \cdot d W_{r}+\sum_{t<\tau_{i}^{t, e} \leq s} c\left(X_{\tau_{i}^{t, e}-, N_{\tau_{i}}^{t, x, e}, N_{\tau_{i}^{t, e}}^{t, e}}^{t, e}, N^{t, e}\right)$,

and we set

$$
u(t, x, e):=\inf \left\{y \in \mathbb{R}: \exists \phi \in \Phi \text { s.t. } Y_{T}^{(t, x, e, y), \phi} \geq g\left(X_{T}^{t, x, e}, N_{T}^{t, e}\right)\right\} .
$$

3. We finally introduce

$$
H_{t}^{\nu}:=\mathcal{E}\left(\int_{t}^{T} \int_{E}\left(\nu_{s}^{\sigma}-1\right) \tilde{\mu}(d \sigma, d s)\right), \nu \in \mathcal{U}
$$

and set

$$
\tilde{u}(t, x, e):=\sup _{\nu \in \mathcal{U}} \mathbb{E}\left[H_{t}^{\nu} \Pi^{t, x}\left(N^{t, e}\right)\right] .
$$

Remark 3.1 It follows from a straightforward extension of (2.16) that $u \geq \tilde{u}$.

Remark 3.2 Fix $(t, x) \in[0, T] \times \mathbb{R}^{d}$ and $\xi \in \mathcal{S}_{0}$. It follows from the admissibility condition (2.3) and standard arguments based on Burkholder-Davis-Gundy's inequality and Gronwall's Lemma that

$$
\mathbb{E}\left[\sup _{t \leq s \leq T}\left|X_{s}^{(t, x), \xi}\right|^{2 \bar{p}}\right]<\infty
$$

Moreover, if $\operatorname{card}\left\{i \geq 1: t<\tau_{i}^{\xi} \leq T\right\} \leq K$ for some real $K>0$, then

$$
\mathbb{E}\left[\sup _{t \leq s \leq T}\left|X_{s}^{(t, x), \xi}\right|^{p}\right] \leq C_{K}^{p}\left(1+|x|^{p}\right)
$$

for some $C_{K}^{p}>0$ which depends only on $b, a, \beta, T, K$ and $p \geq 1$. 


\subsection{Definition of viscosity solutions}

For a function $\varphi$ on $[0, T] \times \mathbb{R}^{d} \times E$ and $(t, x, e) \in[0, T] \times \mathbb{R}^{d} \times E$, we set

$$
\mathcal{G}^{e} \varphi(t, x, e):=\min _{j \in E \backslash\{e\}}(\varphi(t, x, e)-\varphi(t, x+\beta(x, e, j), e, j)+c(x, e, j))
$$

and, for $\varphi \in C^{1,2}\left([0, T] \times \mathbb{R}^{d}\right)$,

$$
\mathcal{L}^{e} \varphi:=\frac{\partial}{\partial t} \varphi+b(\cdot, e)^{\prime} D \varphi+\frac{1}{2} \operatorname{Tr}\left[a a^{\prime}(\cdot, e) D^{2} \varphi\right]+f(\cdot, e)
$$

where $D \varphi$ and $D^{2} \varphi$ are the partial gradient and Hessian matrix of $\varphi$ with respect to its second variable $x$ and ' stands for transposition.

Since the functions $u, \tilde{u}$ and $v$ need not be smooth, we shall appeal to the following classical notion of viscosity solution, see [6] for a general survey on this topic.

Definition 3.1 We say that a lower-semicontinuous (resp. upper-semicontinuous) function $V$ on $[0, T) \times \mathbb{R}^{d} \times E$ is a viscosity supersolution (resp. subsolution) of

$$
\min \{-\mathcal{L} \varphi, \mathcal{G} \varphi\}=0
$$

if, for all $e \in E, \varphi \in C^{1,2}\left([0, T] \times \mathbb{R}^{d}\right)$ and all $(t, x) \in[0, T) \times \mathbb{R}^{d}$ which realizes a local minimum (resp. maximum) of $V(\cdot, e)-\varphi$, we have

$$
\min \left\{-\mathcal{L}^{e} \varphi(t, x), \mathcal{G}^{e} V(t, x, e)\right\} \geq 0 \quad(\text { resp } \leq 0) .
$$

We say that a locally bounded function $w$ is a discontinuous viscosity solution of (3.3) if $w_{*}$ (resp. $\left.w^{*}\right)$ is a supersolution (resp. subsolution) of (3.3) where

$$
\begin{aligned}
& w^{*}(t, x, e):=\limsup _{\left(t^{\prime}, x^{\prime}\right) \rightarrow(t, x), t^{\prime}<T} w\left(t^{\prime}, x^{\prime}, e\right) \\
& w_{*}(t, x, e):=\liminf _{\left(t^{\prime}, x^{\prime}\right) \rightarrow(t, x), t^{\prime}<T} w\left(t^{\prime}, x^{\prime}, e\right), \quad(t, x, e) \in[0, T] \times \mathbb{R}^{d} \times E .
\end{aligned}
$$

We shall prove in the subsequent sections that $v, u$ and $\tilde{u}$ are viscosity solutions of (3.3).

To complete this characterization, we need to provide a suitable boundary condition. In general, we can not expect to have $u(T-, \cdot)=g, \tilde{u}(T-, \cdot)=g$ or $v(T-, \cdot)=g$, and we need to consider the relaxed boundary condition given by the equation

$$
\min \left\{\psi(x, e)-g(x, e), \mathcal{G}^{e} \psi(x, e)\right\}=0, \text { for all }(x, e) \in \mathbb{R}^{d} \times E .
$$

Definition 3.2 We say that a locally bounded map $w$ satisfies the boundary condition $(3.4)$ if $w_{*}(T, \cdot)\left(\right.$ resp. $\left.w^{*}(T, \cdot)\right)$ is a supersolution (resp. subsolution) of (3.4). Here the terms supersolution and subsolution are taken in the classical sens.

If $w$ is a discontinuous viscosity solution of (3.3) and satisfies the boundary condition (3.4), we just say that $w$ is a (discontinuous) viscosity solution of (3.3)-(3.4). We define similarly the notion of super and subsolution of (3.3)-(3.4). 


\subsection{The PDE characterization}

Our main theorem is obtained under the additional assumption that there is a fixed integer $\gamma \geq 1$ such that

H1 : $u^{+}, \tilde{u}^{+}$and $v^{+}$satisfy the growth condition

$$
\sup _{(t, x, e) \in[0, T] \times \mathbb{R}^{d} \times E}|\psi(t, x, e)| /\left(1+|x|^{\gamma}\right)<\infty .
$$

H2 : There is a function $\Lambda$ on $\mathbb{R}^{d} \times E$ satisfying

(i) $\Lambda(\cdot, e) \in C^{2}\left(\mathbb{R}^{d}\right)$ for all $e \in E$,

(ii) $b^{\prime} D \Lambda+\frac{1}{2} \operatorname{Tr}\left[a a^{\prime} D^{2} \Lambda\right] \leq \varrho \Lambda$ on $\mathbb{R}^{d} \times E$, for some $\varrho>0$,

(iii) $\mathcal{G}^{e} \Lambda(x, e) \geq q(x)$ on $\mathbb{R}^{d} \times E$ for some continuous function $q>0$ on $\mathbb{R}^{d}$,

(iv) $\Lambda \geq g^{+}$,

(v) $\Lambda(x, e) /|x|^{\gamma} \rightarrow \infty$ as $|x| \rightarrow \infty$ for all $e \in E$.

This two conditions will only be used to prove and then apply the comparison principle of Proposition 4.8 below. From the technical point of view, they allow to construct a suitable smooth strict supersolution to a slightly modified version of (3.3)-(3.4). We will provide in Section 5 examples of sufficient conditions on the coefficients under which $\mathbf{H 1}$ and $\mathbf{H 2}$ hold.

Theorem 3.1 Assume that $\mathbf{H 1}$ and $\mathbf{H 2}$ hold. Then, $v, u$ and $\tilde{u}$ are continuous on $[0, T) \times \mathbb{R}^{d} \times E$ and coincide with the unique viscosity solution of (3.3)-(3.4) which satisfies the growth condition (3.5).

Proof. It is a consequence of Remark 3.1, Proposition 4.1, Proposition 4.2, Proposition 4.5, Proposition 4.7 and Proposition 4.8 which imply that $\tilde{u} \geq v \geq u \geq \tilde{u}$, see below.

Remark 3.3 Due to the non-standard form of the control problem associated to $u$, it may not satisfy the growth condition (3.5) while $v$ and $\tilde{u}$ do. In this case, we still have $v$ $=\tilde{u}$ under $\mathbf{H 2}$ as a consequence of Proposition 4.1, Proposition 4.2, Proposition 4.7 and Proposition 4.8 below.

In many situations, it is possible to simplify the boundary condition. We explain here how to do it under the natural assumption:

H3: If $\left(e_{i}, x_{i}\right)_{0 \leq i \leq k}, k \geq 1$, is a sequence in $E \times \mathbb{R}^{d}$ such that $x_{i}=x_{i-1}+\beta\left(x_{i-1}, e_{i-1}, e_{i}\right)$ for $1 \leq i \leq k$ and $e_{k}=e_{0}$, then $x_{k}=x_{0}$ and $\sum_{i=1}^{k} c\left(x_{i-1}, e_{i-1}, e_{i}\right)>0$.

The fact that $x_{k}=x_{0}$ just means that we can not make the process $X$ jump without changing the value of the current regime $\xi$. The second assertion is obviously satisfied if $c>0$. Since $c(\cdot, e, e)=1$, it also holds under the triangular condition: $c\left(x, e_{1}, e_{3}\right) \leq$ 
$c\left(x, e_{1}, e_{2}\right)+c\left(x+\beta\left(x, e_{1}, e_{2}\right), e_{2}, e_{3}\right)$ for $e_{1}, e_{2}, e_{3} \in E$. Observe that we can always reduce to this case in the optimal switching problem when $\beta\left(\beta\left(x, e_{1}, e_{2}\right), e_{2}, e_{3}\right)=\beta\left(x, e_{1}, e_{3}\right)$.

Let $\left(G^{n}\right)_{n \geq 0}$ be the sequence of functions on $\mathbb{R}^{d} \times E$ defined by $G^{0}=g$ and

$$
G^{n+1}(x, e)=\max _{j \in E}\left(G^{n}(x+\beta(x, e, j), j)-c(x, e, j) \mathbf{1}_{j \neq e}\right), n \geq 0 .
$$

Since the sequence is non-decreasing, recall (2.9), it admits a limit $G$, taking possibly infinite values.

Proposition 3.1 Assume that there exists a locally bounded supersolution $\psi$ of (3.4). Then, $G$ is locally bounded and is the smallest solution of (3.4).

If moreover $\mathbf{H 3}$ holds, then any subsolution $\varphi$ of (3.4) satisfies $\varphi \leq G$ on $\mathbb{R}^{d} \times E$.

Proof. 1. A simple induction shows that $\psi \geq G^{n}$ for all $n \geq 1$. Passing to the limit in (3.6) and recalling that $G^{0}=g$ concludes the proof of the first assertion.

2. We now assume that $\varphi$ is a subsolution and that $\mathbf{H 3}$ holds. Assume that $\varphi\left(x_{0}, e_{0}\right)-$ $G\left(x_{0}, e_{0}\right)>0$ for some $\left(x_{0}, e_{0}\right) \in \mathbb{R}^{d} \times E$. Then, we can find $e_{1} \in E \backslash\left\{e_{0}\right\}$ such that $\varphi\left(x_{0}, e_{0}\right) \leq \varphi\left(x_{1}, e_{1}\right)-c\left(x_{0}, e_{0}, e_{1}\right)$ where $x_{1}:=x_{0}+\beta\left(x_{0}, e_{0}, e_{1}\right)$. If $\varphi\left(x_{1}, e_{1}\right) \leq G\left(x_{1}, e_{1}\right)$ then we must have $G\left(x_{0}, e_{0}\right)<G\left(x_{1}, e_{1}\right)-c\left(x_{0}, e_{0}, e_{1}\right)$ which contradicts the definition of $G$. By iterating this procedure, we obtain a sequence $\left(e_{i}, x_{i}\right)_{i \geq 0}$ in $E \times \mathbb{R}^{d}$ such that $x_{i}=x_{i-1}+\beta\left(x_{i-1}, e_{i-1}, e_{i}\right)$ for $i \geq 1$ and $\varphi\left(x_{0}, e_{0}\right) \leq \varphi\left(x_{k}, e_{k}\right)-\sum_{i=0}^{k-1} c\left(x_{i}, e_{i}, e_{i+1}\right)$ for $k \geq 1$. Since $E$ is finite, we can choose $k$ such that $e_{k}=e_{0}$ and we obtain a contradiction to $\mathbf{H 3}$.

As a consequence, $G$ is the only solution of (3.4) if $\mathbf{H 3}$ holds.

Corollary 3.1 Assume that $\mathbf{H 3}$ holds and that $w$ is a viscosity solution of (3.3)-(3.4). Then,

$$
\lim _{t \nearrow T} w(t, x, e)=G(x, e) \quad \text { for all }(x, e) \in \mathbb{R}^{d} \times E
$$

\section{Proof of Theorem 3.1}

\subsection{Lower bounds for the value functions}

We first show that $v^{-}, u^{-}$and $\tilde{u}^{-}$have polynomial growth.

Proposition 4.1 The functions $v^{-}, u^{-}$and $\tilde{u}^{-}$satisfy the growth condition (3.5) with $\gamma:=\bar{p}$.

Proof. The result is obtained for $\tilde{u}$ and $u$ by observing that $1 \in \mathcal{U}$, using (2.6), Remark 3.1 and standards estimates. Since $\xi:=e \in \mathcal{S}_{t}(e)$, the bound for $v$ follows from Remark 3.2 and (2.6). 


\subsection{Viscosity properties of the optimal switching problem}

The aim of this section is to prove the following proposition.

Proposition 4.2 Assume that $v$ is locally bounded. Then it is a discontinuous viscosity solution of (3.3)-(3.4).

We split the proof in different propositions. In the rest of this subsection, we always assume that $v$ is locally bounded.

In order to characterize $v$ as a viscosity solution, we shall appeal to the classical dynamic programming principle. Due to the dependence of $\beta$ on $x$, we can not reproduce the proof of Theorem 4.1 in [19].

We first derive some useful properties for the functional

$J(t, x, \xi):=\mathbb{E}\left[g\left(X_{T}^{(t, x), \xi}, \xi_{T}\right)+\int_{t}^{T} f\left(X_{s}^{(t, x), \xi}, \xi_{s}\right) d s-\sum_{t<\tau_{i}^{\xi} \leq T} c\left(X_{\tau_{i}^{\xi}-}^{(t, x), \xi}, \xi_{\tau_{i}^{\xi}-}, \xi_{\tau_{i}^{\xi}}\right)\right]$

defined for $(t, x) \in[0, T] \times \mathbb{R}^{d}$ and $\xi \in \mathcal{S}_{0}$. For $t_{1} \leq t_{2} \leq T$, we set

$$
I_{t_{1}, t_{2}}^{\xi}:=\operatorname{card}\left\{i \geq 1: t_{1}<\tau_{i}^{\xi} \leq t_{2}\right\}
$$

and we denote by $\mathcal{S}_{0}^{b}$ the set of elements $\xi \in \mathcal{S}_{0}$ such that $I_{0, T}^{\xi}$ is essentially bounded. We set $\mathcal{S}_{t}^{b}(e):=\mathcal{S}_{t}(e) \cap \mathcal{S}_{0}^{b}$.

Lemma 4.1 Fix $(t, x, e) \in[0, T) \times \mathbb{R}^{d} \times E$. Then,

(i) $J(\cdot, \xi)$ is continuous for all $\xi \in \mathcal{S}_{0}^{b}$.

(ii) $\sup J(t, x, \xi)=v(t, x, e)$. $\xi \in \mathcal{S}_{t}^{b}(e)$

(iii) $v(\cdot, e)$ is lower semicontinuous.

Proof. (i) Fix $\xi \in \mathcal{S}_{0}^{b}, t_{1} \leq t_{2}, x_{1}, x_{2} \in \mathbb{R}^{d}$ and write $\left(X^{1}, X^{2}\right)$ for $\left(X^{\left(t_{1}, x_{1}\right), \xi}, X^{\left(t_{2}, x_{2}\right), \xi}\right)$. We define the sequence

$$
\vartheta_{i+1}:=\inf \left\{s>\vartheta_{i}: \xi_{s} \neq \xi_{s-}\right\} \text { for } i \geq 0 \text {, with } \vartheta_{0}=t_{2} \text {. }
$$

Standard computations based on Burkholder-Davis-Gundy's inequality, Gronwall's Lemma and the Lipschitz continuity of $b, a, \beta$ shows that

$$
\mathbb{E}\left[\sup _{t_{2} \leq s \leq \vartheta_{i+1} \wedge T}\left|X_{s}^{1}-X_{s}^{2}\right|^{2 \bar{p}}\right] \leq C \mathbb{E}\left[\sup _{t_{2} \leq s \leq \vartheta_{i} \wedge T}\left|X_{s}^{1}-X_{s}^{2}\right|^{2 \bar{p}}\right] \quad i \geq 0,
$$


where $C>0$ denotes a generic constant which may change from line to line. Since $I_{0, T}^{\xi}$ is essentially bounded and $\vartheta_{0}=t_{2}$, we deduce that

$$
\mathbb{E}\left[\sup _{t_{2} \leq s \leq T}\left|X_{s}^{1}-X_{s}^{2}\right|^{2 \bar{p}}\right] \leq C \mathbb{E}\left[\left|X_{t_{2}}^{1}-x_{2}\right|^{2 \bar{p}}\right]
$$

where, by Remark 3.2 and (2.2),

$$
\mathbb{E}\left[\sup _{t_{1} \leq s \leq t_{2}}\left|X_{s}^{1}-x_{1}\right|^{2 \bar{p}}\right] \leq C\left(\left|t_{2}-t_{1}\right|^{\bar{p}}+\mathbb{E}\left[\left|I_{t_{1}, t_{2}}^{\xi}\right|^{2 \bar{p}}\right]\right)
$$

We now fix $(t, x) \in[0, T] \times \mathbb{R}^{d}$ and a sequence $\left(t_{n}, x_{n}\right)_{n \geq 1}$ such that $t_{n} \rightarrow t$ and $x_{n} \rightarrow x$, we write $X$ and $X^{n}$ for $X^{(t, x), \xi}$ and $X^{\left(t_{n}, x_{n}\right), \xi}$. In view of (4.1)-(4.2), we can find a subsequence such that $\sup _{t \vee t_{n} \leq s \leq T}\left|X_{s}^{n}-X_{s}\right| \rightarrow 0 \mathbb{P}$ - a.s. Moreover, if follows from Remark 3.2 that $\mathbb{E}\left[\sup _{t_{n} \leq s \leq T}\left|X_{s}^{n}\right|^{2 \bar{p}}\right]$ is bounded, uniformly in $n \geq 1$. Recalling the growth condition (2.6) and the fact that $I_{0, T}^{\xi}$ is bounded, we deduce that

$$
\liminf _{n \rightarrow \infty} J\left(t_{n}, x_{n}, \xi\right) \geq J(t, x, \xi)-\limsup _{n \rightarrow \infty} \mathbb{E}\left[\left|\sum_{t_{n} \wedge t<\tau_{i}^{\xi} \leq t \vee t_{n}} \Psi\left(\xi_{\tau_{i}^{\xi}}, \xi_{\tau_{i}^{\xi}}\right)\right|\right]=J(t, x, \xi) .
$$

We obtain similarly that $\lim \sup _{n \rightarrow \infty} J\left(t_{n}, x_{n}, \xi\right) \leq J(t, x, \xi)$.

(ii) Fix $\xi \in \mathcal{S}_{0}$ and let $\xi^{k} \in \mathcal{S}_{0}^{b}$ be defined by $\xi_{t}^{k}=\xi_{t \wedge \tau_{k}^{\xi}}, k \geq 1$. Arguing as in Remark 3.2 , we obtain that

$$
\sup _{k \geq 1} \mathbb{E}\left[\sup _{t \leq s \leq T}\left|X_{s}^{(t, x), \xi^{k}}\right|^{2 \bar{p}}\right]<\infty
$$

Moreover, it follows from a similar induction argument as above that

$$
\mathbb{E}\left[\sup _{t \leq s \leq t \vee \tau_{i}^{\xi}}\left|X_{s}^{(t, x), \xi^{k}}-X_{s}^{(t, x), \xi}\right|^{2}\right] \rightarrow 0 \text { for all } i \geq 1 .
$$

After possibly passing to a subsequence, we can then assume that

$$
\sup _{t \leq s \leq t \vee \tau_{i}^{\xi}}\left|X_{s}^{(t, x), \xi^{k}}-X_{s}^{(t, x), \xi}\right| \longrightarrow 0 \mathbb{P}-\text { a.s. } \forall i \geq 1 .
$$

In view of (4.3), we deduce from (2.6), (2.3), (2.7) and the continuity of $g, f$ and $c$ that

$$
\liminf _{k \rightarrow \infty} J\left(t, x, \xi^{k}\right) \geq J(t, x, \xi) .
$$

This proves (ii).

(iii) The last assertion is an immediate consequence of (ii). 
Lemma 4.2 Fix $(t, x, e) \in[0, T] \times \mathbb{R}^{d} \times E$. For all $[t, T]$-valued stopping time $\theta$ and $\xi \in \mathcal{S}_{t}(e)$, we have $v(t, x, e) \geq \mathbb{E}\left[v\left(\theta, X_{\theta}^{(t, x), \xi}, \xi_{\theta}\right)+\int_{t}^{\theta} f\left(X_{s}^{(t, x), \xi}, \xi_{s}\right) d s-\sum_{t<\tau_{i}^{\xi} \leq \theta} c\left(X_{\tau_{i}^{\xi}-}^{(t, x), \xi}, \xi_{\tau_{i}^{\xi}-}, \xi_{\tau_{i}^{\xi}}\right)\right]$.

Proof. Fix $\left(t_{0}, x_{0}, e_{0}\right) \in[0, T) \times \mathbb{R}^{d} \times E$. We write $X^{0, \xi}$ for $X^{\left(t_{0}, x_{0}\right), \xi}, \xi \in \mathcal{S}_{t_{0}}\left(e_{0}\right)$.

1. Fix an open ball $B\left(\left(t_{0}, x_{0}\right), \rho\right), \rho>0$, set $B_{0}:=B\left(\left(t_{0}, x_{0}\right), \rho\right) \cap\left([0, T] \times \mathbb{R}^{d}\right)$, and fix a compact set $\Theta$ such that

$$
\left\{\left(t, x+\beta\left(x, i,[i+j]_{\kappa}\right)\right):(t, x, i, j) \in B_{0} \times E \times E\right\} \subset \Theta \subset[0, T] \times \mathbb{R}^{d},
$$

recall that $\beta$ is continuous and (2.9). Let $\varphi$ be a continuous map on $[0, T] \times \mathbb{R}^{d} \times[0, \kappa]$ such that

$$
\varphi \leq v_{*} \quad \text { on } \Theta \times E .
$$

Let $\left(B_{n}\right)_{n \geq 1}$ be a partition of $\Theta$ and $\left(t_{n}, x_{n}\right)_{n \geq 1}$ be a sequence such that $\left(t_{n}, x_{n}\right) \in B_{n}$ for each $n \geq 1$. It follows from Lemma 4.1 that, for each $n \geq 1$ and $e \in E$, we can find $\xi^{e, n} \in \mathcal{S}_{t_{n}}^{b}(e)$ such that

$$
J\left(t_{n}, x_{n}, \xi^{e, n}\right) \geq v\left(t_{n}, x_{n}, e\right)-\varepsilon / 3,
$$

where $\varepsilon>0$ is a fix parameter. Moreover, by continuity of $\varphi$ and $J(\cdot, \xi)$ for $\xi \in \mathcal{S}_{0}^{b}$, see Lemma 4.1 , we can choose $\left(B_{n}, t_{n}, x_{n}\right)_{n \geq 1}$ in such a way that

$$
\max _{e \in E}\left(\left|\varphi(\cdot, e)-\varphi\left(t_{n}, x_{n}, e\right)\right|+\left|J\left(\cdot, \xi^{e, n}\right)-J\left(t_{n}, x_{n}, \xi^{e, n}\right)\right|\right) \leq \varepsilon / 3 \quad \text { on } B_{n} .
$$

2. Let us set

$$
\vartheta:=\inf \left\{s \in\left[t_{0}, T\right]:\left(s, X_{s}^{0, \xi}\right) \notin B_{0}\right\} \wedge \theta
$$

where $\theta$ is a given stopping time with values in $\left[t_{0}, T\right]$ and $\xi \in \mathcal{S}_{t_{0}}^{b}\left(e_{0}\right)$. We next define $\bar{\xi} \in \mathcal{S}_{t_{0}}^{b}\left(e_{0}\right)$ by

$$
\bar{\xi}_{t}:=\xi_{t} \mathbf{1}_{t \leq \vartheta}+\mathbf{1}_{t>\vartheta}\left(\sum_{e \in E} \sum_{n \geq 1} \xi_{t}^{e, n} \mathbf{1}_{\left\{\left(\vartheta, X_{\vartheta}^{0, \xi}\right) \in B_{n}\right\}} \mathbf{1}_{\xi_{\vartheta}=e}\right) .
$$

Observe that $\bar{\xi}_{\vartheta}=\xi_{\vartheta}$. It follows from (4.4), (4.5), (4.6), (4.7) and the Markov feature of our model that, for all $\xi \in \mathcal{S}_{t_{0}}^{b}\left(e_{0}\right)$,

$$
\begin{aligned}
J\left(t_{0}, x_{0}, \bar{\xi}\right) & \geq \mathbb{E}\left[J\left(\vartheta, X_{\vartheta}^{0, \xi}, \bar{\xi}\right)+\int_{t}^{\vartheta} f\left(X_{s}^{0, \xi}, \xi_{s}\right) d s-\sum_{t<\tau_{i}^{\xi} \leq \vartheta} c\left(X_{\tau_{i}^{\xi}-}^{0, \xi}, \xi_{\tau_{i}^{\xi}-}, \xi_{\tau_{i}^{\xi}}\right)\right] \\
& \geq \mathbb{E}\left[\varphi\left(\vartheta, X_{\vartheta}^{0, \xi}, \xi_{\vartheta}\right)+\int_{t}^{\vartheta} f\left(X_{s}^{0, \xi}, \xi_{s}\right) d s-\sum_{t<\tau_{i}^{\xi} \leq \vartheta} c\left(X_{\tau_{i}^{\xi}-}^{0, \xi}, \xi_{\tau_{i}^{\xi}-}, \xi_{\tau_{i}^{\xi}}\right)\right]-\varepsilon .
\end{aligned}
$$


By arbitrariness of $\varepsilon>0$, this shows that

$$
v\left(t_{0}, x_{0}, e_{0}\right) \geq \mathbb{E}\left[\varphi\left(\vartheta, X_{\vartheta}^{0, \xi}, \xi_{\vartheta}\right)+\int_{t}^{\vartheta} f\left(X_{s}^{0, \xi}, \xi_{s}\right) d s-\sum_{t<\tau_{i}^{\xi} \leq \vartheta} c\left(X_{\tau_{i}^{\xi}-}^{0, \xi}, \xi_{\tau_{i}^{\xi}-}, \xi_{\tau_{i}^{\xi}}\right)\right] .
$$

3. By replacing $\varphi$ by a sequence $\left(\varphi_{k}\right)_{k \geq 1}$ of continuous functions satisfying

$$
\varphi_{k} \leq v_{*} \quad \text { and } \quad \varphi_{k} \nearrow v_{*} \text { on } \Theta \times E
$$

we deduce from (4.8) that, for all $\xi \in \mathcal{S}_{t_{0}}^{b}\left(e_{0}\right)$,

$$
v\left(t_{0}, x_{0}, e_{0}\right) \geq \mathbb{E}\left[v_{*}\left(\vartheta, X_{\vartheta}^{0, \xi}, \xi_{\vartheta}\right)+\int_{t}^{\vartheta} f\left(X_{s}^{0, \xi}, \xi_{s}\right) d s-\sum_{t<\tau_{i}^{\xi} \leq \vartheta} c\left(X_{\tau_{i}^{\xi}-}^{0, \xi}, \xi_{\tau_{i}^{\xi}-}, \xi_{\tau_{i}^{\xi}}\right)\right] .
$$

Letting $\rho$ go to infinity in the definition of $B_{0}$, using Remark 3.2, the growth assumption (2.6), the fact that $I_{0, T}^{\xi}$ is bounded, (2.7) and Proposition 4.1, we deduce from the above inequality that

$v\left(t_{0}, x_{0}, e_{0}\right) \geq \mathbb{E}\left[v_{*}\left(\theta, X_{\theta}^{0, \xi}, \xi_{\theta}\right)+\int_{t}^{\theta} f\left(X_{s}^{0, \xi}, \xi_{s}\right) d s-\sum_{t<\tau_{i}^{\xi} \leq \theta} c\left(X_{\tau_{i}^{\xi}-}^{0, \xi}, \xi_{\tau_{i}^{\xi}-}, \xi_{\tau_{i}^{\xi}}\right)\right]$.

In view of Proposition 4.1, it then follows from the same arguments as in (ii) of the proof of Lemma 4.1 that the above inequality actually holds for all $\xi \in \mathcal{S}_{t}(e)$. The proof is concluded by using (iii) of Lemma 4.1.

Remark 4.1 The inequality

$$
v(t, x, e) \leq \sup _{\xi \in \mathcal{S}_{t}(e)} \mathbb{E}\left[v\left(\theta, X_{\theta}^{(t, x), \xi}, \xi_{\theta}\right)+\int_{t}^{\theta} f\left(X_{s}^{(t, x), \xi}, \xi_{s}\right) d s-\sum_{t<\tau_{i}^{\xi} \leq \theta} c\left(X_{\tau_{i}^{\xi}-}^{(t, x), \xi}, \xi_{\tau_{i}^{\xi}-}, \xi_{\tau_{i}^{\xi}}\right)\right]
$$

follows from the Markov feature of our model. In view of Lemma 4.2, we therefore retrieve the usual dynamic programming principle.

Proposition 4.3 The function $v_{*}$ is a viscosity supersolution of (3.3)-(3.4).

Proof. The proof is standard and we only sketch it. Fix $\left(t_{0}, x_{0}, e_{0}\right) \in[0, T] \times \mathbb{R}^{d} \times E$ and let $\left(t_{k}, x_{k}\right)_{k \geq 1}$ be a sequence in $[0, T) \times \mathbb{R}^{d}$ such that

$$
\left(t_{k}, x_{k}\right) \longrightarrow\left(t_{0}, x_{0}\right) \text { and } v\left(t_{k}, x_{k}, e_{0}\right) \longrightarrow v_{*}\left(t_{0}, x_{0}, e_{0}\right) \text { as } k \longrightarrow \infty \text {. }
$$

Given $\xi^{k} \in \mathcal{S}_{t_{k}}\left(e_{0}\right)$ to be chosen later, we write $X^{k}$ and $\tau_{i}^{k}$ for $X^{\left(t_{k}, x_{k}\right), \xi^{k}}$ and $\tau_{i}^{\xi^{k}}$. 
1. We first assume that $t_{0}=T$. By taking $\xi^{k}=e_{0} \in \mathcal{S}_{t_{k}}\left(e_{0}\right)$, we deduce from the definition of $v$ that

$$
v\left(t_{k}, x_{k}, e_{0}\right) \geq \mathbb{E}\left[g\left(X_{T}^{k}, e_{0}\right)+\int_{t_{k}}^{T} f\left(X_{s}^{k}, e_{0}\right) d s\right] .
$$

Using standard estimates on $X^{k}$, we deduce from our continuity and growth assumptions on $f, g$ that

$$
v_{*}\left(T, x_{0}, e_{0}\right) \geq \liminf _{k \rightarrow \infty} \mathbb{E}\left[\left(g\left(X_{T}^{k}, e_{0}\right)+\int_{t_{k}}^{T} f\left(X_{s}^{k}, e_{0}\right) d s\right)\right]=g\left(x_{0}, e_{0}\right) .
$$

We now fix $j \in E$, set $\tau_{k}:=\left(T+t_{k}\right) / 2$ and $\xi^{k}:=\left(e_{0} \mathbf{1}_{t<\tau_{k}}+j \mathbf{1}_{t \geq \tau_{k}}\right)_{t \leq T} \in \mathcal{S}_{t_{k}}\left(e_{0}\right)$. By Lemma 4.2

$v\left(t_{k}, x_{k}, e_{0}\right) \geq \mathbb{E}\left[v_{*}\left(\tau_{k}, X_{\tau_{k}-}^{k}+\beta\left(X_{\tau_{k}-}^{k}, e_{0}, j\right), j\right)+\int_{t_{k}}^{\tau_{k}} f\left(X_{s}^{k}, e_{0}\right) d s-c\left(X_{\tau_{k}-}^{k}, e_{0}, j\right)\right]$.

Sending $k \rightarrow \infty$, using Proposition 4.1 and standard estimates shows that

$$
v_{*}\left(T, x_{0}, e_{0}\right) \geq v_{*}\left(T, x_{0}+\beta\left(x_{0}, e_{0}, j\right), j\right)-c\left(x_{0}, e_{0}, j\right) .
$$

2. We now fix $t_{0}<T$. By considering the sequence of controls $\xi^{k}=\left(e_{0} \mathbf{1}_{t<\tau_{k}}+\right.$ $\left.j \mathbf{1}_{\tau_{k} \leq t}\right)_{t \leq T} \in \mathcal{S}_{t_{k}}\left(e_{0}\right)$ where $\tau_{k}:=t_{k}+k^{-1}, j \in E$, using Lemma 4.2 and arguing as above, we obtain

$$
v_{*}\left(t_{0}, x_{0}, e_{0}\right)=\lim _{k \rightarrow \infty} v\left(t_{k}, x_{k}, e_{0}\right) \geq v_{*}\left(t_{0}, x_{0}+\beta\left(x_{0}, e_{0}, j\right), j\right)-c\left(x_{0}, e_{0}, j\right) .
$$

The fact that $v_{*}$ is a supersolution of $-\mathcal{L} \varphi=0$ is obtained by considering constant control processes, Lemma 4.2 and using standard arguments (see the proof of Proposition 4.7 below for a detailed proof in a more complex case).

Proposition 4.4 The function $v^{*}$ is a viscosity subsolution of (3.3)-(3.4).

Proof. 1. We first consider the viscosity property. We argue by contradiction. Fix $\left(t_{0}, x_{0}, e_{0}\right) \in[0, T) \times \mathbb{R}^{d} \times E$ and $\varphi \in C^{1,2}\left([0, T] \times \mathbb{R}^{d}\right)$ such that

$$
0=\left(v^{*}\left(\cdot, e_{0}\right)-\varphi\right)\left(t_{0}, x_{0}\right)=\max _{[0, T] \times \mathbb{R}^{d}}\left(v^{*}\left(\cdot, e_{0}\right)-\varphi\right)
$$

and assume that

$$
\min \left\{-\mathcal{L}^{e_{0}} \varphi\left(t_{0}, x_{0}\right), \mathcal{G}^{e_{0}} v^{*}\left(t_{0}, x_{0}, e_{0}\right)\right\}=: 2 \varepsilon>0 .
$$

Since $\varphi\left(t_{0}, x_{0}\right)=v^{*}\left(t_{0}, x_{0}, e_{0}\right)$, it follows from the upper-semicontinuity of $v^{*}$ that we can find $\delta \in\left(0, T-t_{0}\right)$ for which

$$
\min \left\{-\mathcal{L}^{e_{0}} \varphi, \min _{j \in E \backslash\left\{e_{0}\right\}}\left(\varphi-v^{*}\left(\cdot, \cdot+\beta\left(\cdot, e_{0}, j\right), j\right)+c\left(\cdot, e_{0}, j\right)\right)\right\} \geq \varepsilon>0
$$


on $B:=B\left(t_{0}, \delta\right) \times B\left(x_{0}, \delta\right)$. Observe that we can assume, without loss of generality, that $\left(t_{0}, x_{0}\right)$ achieves a strict local maximum so that

$$
\sup _{\partial_{p} B\left(\left(t_{0}, x_{0}\right), \delta\right)}\left(v^{*}\left(\cdot, e_{0}\right)-\varphi\right)=:-\zeta<0,
$$

where $\partial_{p} B=\left[t_{0}, t_{0}+\delta\right] \times \partial B\left(x_{0}, \delta\right) \cup\left\{t_{0}+\delta\right\} \times B\left(x_{0}, \delta\right)$. Let $\left(t_{k}, x_{k}\right)_{k \geq 1}$ be a sequence in $[0, T) \times \mathbb{R}^{d}$ satisfying

$$
\left(t_{k}, x_{k}\right) \longrightarrow\left(t_{0}, x_{0}\right) \text { and } v\left(t_{k}, x_{k}, e_{0}\right) \longrightarrow v^{*}\left(t_{0}, x_{0}, e_{0}\right) \text { as } k \longrightarrow \infty
$$

so that

$$
v\left(t_{k}, x_{k}, e_{0}\right)-\varphi\left(t_{k}, x_{k}\right) \longrightarrow 0 \quad \text { as } k \rightarrow \infty
$$

Let $\xi^{k}$ be a $k^{-1}$-optimal control for $v\left(t_{k}, x_{k}, e_{0}\right)$, i.e. such that $v\left(t_{k}, x_{k}, e_{0}\right)$ is bounded from above by

$$
\mathbb{E}\left[g\left(X_{T}^{k}, \xi_{T}\right)+\int_{t_{k}}^{T} f\left(X_{s}^{k}, \xi_{s}^{k}\right) d s-\sum_{t_{k}<\tau_{i}^{k} \leq T} c\left(X_{\tau_{i}^{k-}}^{k}, \xi_{\tau_{i}^{k}-}^{k}, \xi_{\tau_{i}^{k}}^{k}\right)\right]+k^{-1}
$$

where $X^{k}$ denotes $X^{\left(t_{k}, x_{k}\right), \xi^{k}}$ and $\tau_{i}^{k}$ stands for $\tau_{i}^{\xi^{k}}$. Set $\vartheta^{k}:=\inf \left\{s>t_{k}: \xi_{k} \neq e_{0}\right\}$, $\theta^{k}:=\inf \left\{s \geq t_{k}:\left(s, X_{s}^{k}\right) \notin B\right\} \wedge \vartheta^{k}$ and $\mathcal{E}_{k}:=\xi_{\theta^{k}}^{k}$. By taking the conditional expectation with respect to $\mathcal{F}_{\theta^{k}}$ in the above expression and using the Markov property of $\left(X^{k}, \xi^{k}\right)$, we get

$$
\begin{aligned}
v\left(t_{k}, x_{k}, e_{0}\right) & \leq \mathbb{E}\left[v\left(\theta^{k}, X_{\theta^{k}-}^{k}+\beta\left(X_{\theta^{k}-}^{k}, e_{0}, \mathcal{E}_{k}\right), \mathcal{E}_{k}\right)\right] \\
& +\mathbb{E}\left[\int_{t_{k}}^{\theta^{k}} f\left(X_{s}^{k}, e_{0}\right) d s-c\left(X_{\theta^{k}-}^{k}, e_{0}, \mathcal{E}_{k}\right) \mathbf{1}_{\theta^{k}=\vartheta^{k}}\right]+k^{-1},
\end{aligned}
$$

recall that $\beta(\cdot, e, e)=0$ for $e \in E$. On the other hand, applying Itô's Lemma to $\varphi$ and using (4.9) and (4.10) leads to

$$
\begin{aligned}
\varphi\left(t_{k}, x_{k}\right) & \geq \mathbb{E}\left[\varphi\left(\theta^{k}, X_{\theta^{k}-}^{k}\right)+\int_{t_{k}}^{\theta^{k}} f\left(X_{s}^{k}, e_{0}\right) d s\right] \\
& \geq \mathbb{E}\left[v^{*}\left(\theta^{k}, X_{\theta^{k}-}^{k}+\beta\left(X_{\theta^{k}-}^{k}, e_{0}, \mathcal{E}_{k}\right), \mathcal{E}_{k}\right)\right] \\
& +\mathbb{E}\left[\int_{t_{k}}^{\theta^{k}} f\left(X_{s}^{k}, e_{0}\right) d s-c\left(X_{\theta^{k}-}^{k}, e_{0}, \mathcal{E}_{k}\right) \mathbf{1}_{\theta^{k}=\vartheta^{k}}\right]+\varepsilon \wedge \zeta .
\end{aligned}
$$

In view of (4.11) and (4.12), this leads to a contradiction for $k$ large enough.

2. It remains to show that

$$
\min \left\{\left(v^{*}-g\right)\left(T, x_{0}, e_{0}\right), \mathcal{G}^{e_{0}} v^{*}\left(T, x_{0}, e_{0}\right)\right\} \leq 0
$$


We argue by contradiction and assume that

$$
\min \left\{\left(v^{*}-g\right)\left(T, x_{0}, e_{0}\right), \mathcal{G}^{e_{0}} v^{*}\left(T, x_{0}, e_{0}\right)\right\}=: 2 \varepsilon>0
$$

Let $\left(t_{k}, x_{k}\right)_{k \geq 1}$ be a sequence in $[0, T] \times \mathbb{R}^{d}$ satisfying

$$
\left(t_{k}, x_{k}\right) \longrightarrow\left(t_{0}, x_{0}\right) \text { and } v\left(t_{k}, x_{k}, e_{0}\right) \rightarrow v^{*}\left(t_{0}, x_{0}, e_{0}\right) \text { as } k \longrightarrow \infty
$$

Under the above assumption, we can find a sequence of smooth functions $\left(\varphi^{n}\right)_{n \geq 0}$ on $[0, T] \times \mathbb{R}^{d}$ such that $\varphi^{n} \rightarrow v^{*}\left(\cdot, e_{0}\right)$ and

$$
\min \left\{\varphi^{n}-g\left(\cdot, e_{0}\right), \min _{j \in E \backslash\left\{e_{0}\right\}}\left(\varphi^{n}-v^{*}\left(\cdot, \cdot+\beta\left(\cdot, e_{0}, j\right), j\right)+c\left(\cdot, e_{0}, j\right)\right)\right\} \geq \varepsilon
$$

on some neighborhood $B_{n}$ of $\left(T, x_{0}\right)$. After possibly passing to a subsequence of $\left(t_{k}, x_{k}\right)_{k \geq 1}$, we can then assume that it holds on $B_{n}^{k}:=\left[t_{k}, T\right] \times B\left(x_{k}, \delta_{n}^{k}\right)$ for some sufficiently small $\delta_{n}^{k} \in(0,1]$ such that $B_{n}^{k} \subset B_{n}$. Since $v^{*}$ is locally bounded, there is some $\zeta>0$ such that $\left|v^{*}\right| \leq \zeta$ on $B_{n}$. We can then assume that $\varphi^{n} \geq-2 \zeta$ on $B_{n}$. Let us define $\tilde{\varphi}_{k}^{n}$ by

$$
\tilde{\varphi}_{k}^{n}(t, x):=\varphi^{n}(t, x)+4 \zeta\left|x-x_{k}\right|^{2} /\left(\delta_{n}^{k}\right)^{2}+\sqrt{T-t},
$$

and observe that

$$
\left(v^{*}\left(\cdot, e_{0}\right)-\tilde{\varphi}_{k}^{n}\right)(t, x) \leq-\zeta<0 \quad \text { for }(t, x) \in\left[t_{k}, T\right] \times \partial B\left(x_{k}, \delta_{n}^{k}\right) .
$$

Since $(\partial / \partial t)(\sqrt{T-t}) \rightarrow-\infty$ as $t \rightarrow T$, we can choose $t_{k}$ large enough in front of $\delta_{n}^{k}$ and the derivatives of $\varphi^{n}$ to ensure that

$$
-\mathcal{L}^{e_{0}} \tilde{\varphi}_{k}^{n} \geq 0 \quad \text { on } B_{n}^{k}
$$

Let $X^{k}, \xi^{k}$ and $\vartheta^{k}$ be defined as in Step 1 and set $\theta_{n}^{k}:=\inf \left\{s \geq t_{k}:\left(s, X_{s}^{k}\right) \notin B_{n}^{k}\right\} \wedge \vartheta^{k}$. Using Itô's Lemma on $\tilde{\varphi}_{k}^{n}$ together with (4.15), (4.16) and (4.17), we obtain that

$$
\begin{aligned}
\tilde{\varphi}_{k}^{n}\left(t_{k}, x_{k}\right) \geq & \mathbb{E}\left[\left(v^{*}\left(\theta^{k}, X_{\theta_{n}^{k}-}^{k}+\beta\left(X_{\theta_{n}^{k-}}^{k}, e_{0}, \mathcal{E}_{k}\right), \mathcal{E}_{k}\right)-c\left(X_{\theta_{n}^{k-}}^{k}, e_{0}, \mathcal{E}_{k}\right)\right) \mathbf{1}_{\vartheta^{k} \leq \theta_{n}^{k}}\right] \\
& +\mathbb{E}\left[\left(v^{*}\left(\theta^{k}, X_{\theta_{n}^{k}}^{k}, e_{0}\right) \mathbf{1}_{\theta_{n}^{k}<T}+g\left(X_{T}^{k}, e_{0}\right) \mathbf{1}_{\theta_{n}^{k}=T}\right) \mathbf{1}_{\theta_{n}^{k}<\vartheta^{k}}\right] \\
& +\mathbb{E}\left[\int_{t_{k}}^{\theta_{n}^{k}} f\left(X_{s}^{k}, e_{0}\right) d s\right]+\varepsilon \wedge \zeta .
\end{aligned}
$$

Since $v(T, \cdot)=g,(4.12)$ implies that

$$
\varphi^{n}\left(t_{k}, x_{k}\right)+\sqrt{T-t_{k}}=\tilde{\varphi}_{k}^{n}\left(t_{k}, x_{k}\right) \geq v\left(t_{k}, x_{k}, e_{0}\right)+\varepsilon \wedge \zeta-k^{-1} .
$$

We then obtain a contradiction by sending $k \rightarrow \infty$ and taking $n$ large enough, recall (4.14). 


\subsection{Viscosity properties for the stochastic target problem}

We first prove the following proposition.

Proposition 4.5 Assume that $u$ is locally bounded. Then $u^{*}$ is a viscosity subsolution of (3.3)-(3.4).

It essentially follows from the general results of [2] up to two differences. First, our control process is not bounded. But it only appears in a martingale term and is therefore easily handled. Second, the notion of viscosity solution used in [2] is (a-priori) slightly different from the one of Definition 3.1 (in our setting they are indeed equivalent, this is easily understood from the arguments below). For sake of clarity and completeness, we therefore provide a new proof which directly fits in our framework.

One could also prove that $u_{*}$ is a supersolution of (3.3)-(3.4) by combining the argument of [2] and those used in the proof of Proposition 4.7 below. Since it is not of direct use, we omit it.

Following [2] and [16], we rely on the direct dynamic programming principle of [17]. In this paper, we only need one part, which can be written as follows.

Proposition 4.6 Fix $(t, x, e) \in[0, T] \times \mathbb{R}^{d} \times E$. Set $y<u(t, x, e)$ and let $\theta$ be a stopping time with values in $[t, T]$. Then, for all $\phi \in \Phi$,

$$
\mathbb{P}\left[Y_{\theta}^{(t, x, e, y), \phi}>u\left(\theta, X_{\theta}^{t, x, e}, N_{\theta}^{t, e}\right)\right]<1 .
$$

Proof of Proposition 4.5. 1. We start with the subsolution property in the domain. We argue by contradiction. Fix $\left(t_{0}, x_{0}, e_{0}\right) \in[0, T) \times \mathbb{R}^{d} \times \mathbb{E}$ and let $\varphi \in C^{1,2}\left([0, T] \times \mathbb{R}^{d}\right)$ be such that

$$
0=\left(u^{*}\left(\cdot, e_{0}\right)-\varphi\right)\left(t_{0}, x_{0}\right)=\max _{[0, T] \times \mathbb{R}^{d}}\left(u^{*}\left(\cdot, e_{0}\right)-\varphi\right)
$$

and assume that

$$
\min \left\{-\mathcal{L}^{e_{0}} \varphi\left(t_{0}, x_{0}\right), \mathcal{G}^{e_{0}} u^{*}\left(t_{0}, x_{0}, e_{0}\right)\right\}=: 2 \varepsilon>0 .
$$

Since $\varphi\left(t_{0}, x_{0}\right)=u^{*}\left(t_{0}, x_{0}, e_{0}\right)$, we can find $\delta \in\left(0, T-t_{0}\right)$ such that

$$
\min \left\{-\mathcal{L}^{e_{0}} \varphi, \min _{j \in E \backslash\left\{e_{0}\right\}}\left(\varphi-u^{*}\left(\cdot, \cdot+\beta\left(\cdot, e_{0}, j\right), j\right)+c\left(\cdot, e_{0}, j\right)\right)\right\} \geq \varepsilon>0
$$

on $B:=B\left(t_{0}, \delta\right) \times B\left(x_{0}, \delta\right)$. Observe that we can assume, without loss of generality, that $\left(t_{0}, x_{0}\right)$ achieves a strict local maximum so that

$$
\sup _{\partial_{p} B\left(\left(t_{0}, x_{0}\right), \delta\right)}\left(u^{*}\left(\cdot, e_{0}\right)-\varphi\right)=:-\zeta<0
$$


where $\partial_{p} B=\left[t_{0}, t_{0}+\delta\right) \times \partial B\left(x_{0}, \delta\right) \cup\left\{t_{0}+\delta\right\} \times B\left(x_{0}, \delta\right)$.

Let $\left(t_{k}, x_{k}\right)_{k \geq 1}$ be a sequence in $[0, T] \times \mathbb{R}^{d}$ satisfying

$$
\left(t_{k}, x_{k}\right) \longrightarrow\left(t_{0}, x_{0}\right) \text { and } u\left(t_{k}, x_{k}, e_{0}\right) \longrightarrow u^{*}\left(t_{0}, x_{0}, e_{0}\right) \text { as } k \longrightarrow \infty
$$

and notice that

$$
\eta_{k}:=\varphi\left(t_{k}, x_{k}\right)-u\left(t_{k}, x_{k}, e_{0}\right) \longrightarrow 0 \quad \text { as } k \longrightarrow \infty .
$$

Set $y_{k}:=u\left(t_{k}, x_{k}, e_{0}\right)-k^{-1}$ and denote $N^{k}$ for $N^{t_{k}, e_{0}}$ and $\left(X^{k}, Y^{k}\right)$ for $\left(X^{t_{k}, x_{k}, e_{0}}\right.$, $\left.Y^{\left(t_{k}, x_{k}, e_{0}, y_{k}\right), \phi_{k}}\right)$ where $\phi_{k}$ is the (bounded) feedback control process $\phi^{k}:=D \varphi\left(\cdot, X^{k}(\cdot-)\right.$, $\left.N^{k}(\cdot-)\right)$. Set $\vartheta^{k}:=\inf \left\{s \geq t_{k}: N_{s}^{k} \neq e_{0}\right\}, \theta^{k}:=\inf \left\{s \geq t_{k}:\left(s, X_{s}^{k}\right) \notin B\right\} \wedge \vartheta^{k}$. Since $N_{\theta_{k}-}^{k}=e_{0}$, if follows from (4.18) and (4.19) that

$$
\begin{aligned}
Y_{\theta^{k}}^{k} & =y_{k}-\int_{t_{k}}^{\theta^{k}} f\left(X_{s}^{k}, N_{s}^{k}\right) d s+\int_{t_{k}}^{\theta^{k}} \phi_{s}^{k} \cdot d W_{s}+c\left(\theta_{k}, X_{\theta_{k}-}^{k}, N_{\theta_{k}-}^{k}, N_{\theta_{k}}^{k}\right) \mathbf{1}_{\theta_{k}=\vartheta_{k}} \\
& \geq-k^{-1}-\eta_{k}+\varphi\left(\theta_{k}, X_{\theta_{k}-}^{k}\right)+c\left(\theta_{k}, X_{\theta_{k}-}^{k}, N_{\theta_{k}-}^{k}, N_{\theta_{k}}^{k}\right) \mathbf{1}_{\theta_{k}=\vartheta_{k}} \\
& \geq-k^{-1}-\eta_{k}+u^{*}\left(\theta_{k}, X_{\theta_{k}}^{k}, N_{\theta_{k}}^{k}\right)+\zeta \mathbf{1}_{\theta_{k}<\vartheta_{k}}+\varepsilon \mathbf{1}_{\theta_{k}=\vartheta_{k}} .
\end{aligned}
$$

Since $y_{k}<u\left(t_{k}, x_{k}, e_{0}\right)$ and $\eta_{k} \rightarrow 0$ this contradicts Proposition 4.6 for $k$ large enough. 2. The boundary condition is obtained by combining the arguments of the first step with the arguments of the second step of the proof of Proposition 4.4.

We now turn to $\tilde{u}$.

Proposition 4.7 Assume that $\tilde{u}$ is locally bounded. Then it is a discontinuous viscosity solution of (3.3)-(3.4).

As in Section 4.2, we use a dynamic programming principle.

Lemma 4.3 Fix $(t, x, e) \in[0, T) \times \mathbb{R}^{d} \times E$ and let $\theta$ be $a[t, T]$-valued stopping time. Then, $\tilde{u}(t, x, e)$ is bounded from below by

$$
\sup _{\nu \in \mathcal{U}} \mathbb{E}\left[H_{t}^{\nu}\left(\tilde{u}\left(\theta, X_{\theta}^{t, x, e}, N_{\theta}^{t, e}\right)+\int_{t}^{\theta} f\left(X_{s}^{t, x, e}, N_{s}^{t, e}\right) d s-\sum_{t<\tau_{i}^{t, e} \leq \theta} c\left(X_{\tau_{i}^{t, e}-}^{t, x, e}, N_{\tau_{i}^{t, e}-}^{t, e}, N_{\tau_{i}^{t, e}}^{t, e}\right)\right] .\right.
$$

Proof. The continuity of the map

$$
(t, x) \mapsto \mathbb{E}\left[H_{t}^{\nu} \Pi^{t, x}\left(N^{t, e}\right)\right], \quad(\nu, e) \in \mathcal{U} \times E,
$$

is easily checked because each element of $\mathcal{U}$ is essentially bounded. This implies that $\tilde{u}$ is lower semicontinuous. The result is then obtained by arguing as in the proof of Lemma 4.2 . 
Proof of Proposition 4.7. The subsolution property follows from similar arguments as in the proof of Proposition 4.4, the proof is therefore omitted. We now prove the supersolution property inside the domain. Fix $\left(t_{0}, x_{0}, e_{0}\right) \in[0, T) \times \mathbb{R}^{d} \times \mathbb{E}$ and $\varphi \in$ $C^{1,2}\left([0, T) \times \mathbb{R}^{d}\right)$ such that

$$
0=\left(\tilde{u}_{*}-\varphi\right)\left(t_{0}, x_{0}, e_{0}\right)=\min _{[0, T] \times \mathbb{R}^{d}}\left(\tilde{u}_{*}\left(\cdot, e_{0}\right)-\varphi\right) .
$$

Fix an open ball $B\left(\left(t_{0}, x_{0}\right), \rho\right)$ with $\rho>0$ and a compact set $\Theta$ such that

$$
\left\{\left(t, x+\beta\left(x, e_{0},\left[e_{0}+j\right]_{\kappa}\right)\right):(t, x, j) \in B\left(\left(t_{0}, x_{0}\right), \rho\right) \times E\right\} \subset \Theta,
$$

recall (2.9). For $j \in E$, we consider a sequence of smooth functions $\left(\varphi_{n}^{j}\right)_{n \geq 1}$ on $[0, T] \times \mathbb{R}^{d}$ such that

$$
\varphi_{n}^{j}\left(t_{0}, x_{0}+\beta\left(x_{0}, e_{0}, j\right)\right) \rightarrow \tilde{u}_{*}\left(t_{0}, x_{0}+\beta\left(x_{0}, e_{0}, j\right), j\right) \text { and } \sup _{(t, x) \in \Theta}\left(\varphi_{n}^{j}-\tilde{u}_{*}(\cdot, j)\right) \leq 0
$$

recall that $\tilde{u}$ is locally bounded. We then define $\tilde{\varphi}_{n}$ on $[0, T] \times \mathbb{R}^{d} \times E$ by

$$
\tilde{\varphi}_{n}(t, x, j):=\varphi(t, x) \mathbf{1}_{j=e_{0}}+\varphi_{n}^{j}(t, x) \mathbf{1}_{j \neq e_{0}} .
$$

1. Let $\left(t_{k}, x_{k}\right)_{k \geq 1}$ be a sequence in $[0, T) \times \mathbb{R}^{d}$ satisfying

$$
\left(t_{k}, x_{k}\right) \longrightarrow\left(t_{0}, x_{0}\right) \text { and } \tilde{u}\left(t_{k}, x_{k}, e_{0}\right) \rightarrow \tilde{u}_{*}\left(t_{0}, x_{0}, e_{0}\right) \text { as } k \rightarrow \infty
$$

We write $\left(X^{k}, N^{k}\right)$ for $\left(X^{t_{k}, x_{k}, e_{k}}, N^{t_{k}, e_{k}}\right)$, set $\eta_{k}:=\tilde{u}\left(t_{k}, x_{k}, e_{0}\right)-\varphi\left(t_{k}, x_{k}\right)$ and introduce the sequence

$$
\begin{aligned}
\vartheta_{k} & :=\inf \left\{s \in\left[t_{k}, T\right]:\left(s, X_{s}^{k}\right) \notin B\left(\left(t_{0}, x_{0}\right), \rho\right)\right\} \\
\theta_{k} & :=\vartheta_{k} \wedge\left(t_{k}+h_{k}\right)
\end{aligned}
$$

where $h_{k}$ is defined as follows: If the set $\left\{k \geq 0: \eta_{k}=0\right\}$ is finite, then we may assume that $\eta_{k} \neq 0$ for all $k$ and we set $h_{k}:=\sqrt{\eta_{k}}$. If the set $\left\{k \geq 0: \eta_{k}=0\right\}$ is not finite, then we may assume that $\eta_{k}=0$ for all $k$ and we set $h_{k}:=1 / k$. Notice that in both cases

$$
\lim _{k \rightarrow \infty} \eta_{k} / h_{k}=0
$$

2. We now construct a suitable element of $\mathcal{U}$. For $e \in E$, we set

$$
\chi_{n}(e):=\left\{\begin{array}{cc}
1 & \text { if } \quad \mathcal{H}^{e} \tilde{\varphi}_{n}\left(t_{0}, x_{0}, e_{0}\right) \leq 0 \\
0 & \text { otherwise }
\end{array}\right.
$$

where, for $(t, x, j, e) \in[0, T] \times \mathbb{R}^{d} \times E^{2}$,

$$
\mathcal{H}^{e} \tilde{\varphi}_{n}(t, x, j):=\tilde{\varphi}_{n}(t, x, j)-\tilde{\varphi}_{n}(t, x+\beta(x, j, e), e)+c(x, j, e)
$$


Given $m>0$, we next define the element $\nu^{n, k}$ of $\mathcal{U}$ by

$$
\nu^{n, k}:=\left(m \chi_{n}(j)+m^{-1}\right)_{j \leq \kappa} \mathbf{1}_{\left(t_{k}, \theta_{k}^{n}\right]} \cdot
$$

We denote by $\mathbb{Q}^{n, k}$ the equivalent measure defined by $d \mathbb{Q}^{n, k} / d \mathbb{P}:=H_{t_{k}}^{\nu^{n, k}}$ and by $\mathbb{E}^{n, k}$ the expectation operator under $\mathbb{Q}^{n, k}$.

3. By Lemma $4.3,(4.20)$ and $(4.21)$, we obtain

$\eta_{k} \geq \mathbb{E}^{n, k}\left[\int_{t_{k}}^{\theta_{k}} \mathcal{L}^{N_{s}^{k}} \tilde{\varphi}_{n}\left(s, X_{s}^{k}, N_{s}^{k}\right) d s-\int_{t_{k}}^{\theta_{k}} \sum_{e \in E}\left(m \chi_{n}(e)+m^{-1}\right) \mathcal{H}^{e} \tilde{\varphi}_{n}\left(s, X_{s-}^{k}, N_{s-}^{k}\right) d s\right]$,

see [5]. Using standard arguments, see e.g. [2], one easily checks that

$$
\mathbb{E}\left[\sup _{t_{k} \leq s \leq t_{k}+h_{k}}\left(\left|X_{s}^{k}-x_{0}\right|^{2}+\left|N_{s}^{k}-e_{0}\right|^{2}+\left|H_{t_{k}}^{\nu^{n, k}}-1\right|^{2}\right)\right] \rightarrow 0 \text { as } k \rightarrow \infty .
$$

In particular, this implies that

$$
\liminf _{k \rightarrow \infty} \vartheta_{k}>t_{0}
$$

see [15]. Moreover, observe that for a function $\psi$ on $[0, T] \times \mathbb{R}^{d} \times E$ which is 1-Lipschitz in its two first variables, uniformly in the last one, we have

$$
\begin{aligned}
& \int_{t_{k}}^{t_{k}+h_{k}}\left|\psi\left(s, X_{s}^{k}, N_{s}^{k}\right)-\psi\left(t_{0}, x_{0}, e_{0}\right)\right| d s \\
& \leq\left(h_{k}\right)^{2}+h_{k}\left|t_{k}-t_{0}\right|+h_{k} \sup _{t_{k} \leq s \leq t_{k}+h_{k}}\left|X_{s}^{k}-x_{0}\right|^{2}+h_{k} \sup _{t_{k} \leq s \leq t_{k}+h_{k}}\left|N_{s}^{k}-e_{0}\right|^{2} .
\end{aligned}
$$

Since $N^{k}$ takes values in $E$, we deduce from the previous inequality, (4.24) and (4.25) that, up to a subsequence,

$$
\frac{1}{h_{k}} \int_{t_{k}}^{t_{k}+h_{k}}\left|\psi\left(s, X_{s}^{k}, N_{s}^{k}\right)-\psi\left(t_{0}, x_{0}, e_{0}\right)\right| d s \quad \rightarrow \quad 0 \mathbb{P}-\text { a.s. as } k \rightarrow \infty .
$$

Recalling (4.22), using the dominated convergence theorem and applying the above result in (4.23), we deduce that

$$
0 \geq \mathcal{L}^{e_{0}} \tilde{\varphi}_{n}\left(t_{0}, x_{0}, e_{0}\right)-\sum_{e \in E}\left(m \chi_{n}(e)+m^{-1}\right) \mathcal{H}^{e} \tilde{\varphi}_{n}\left(t_{0}, x_{0}, e_{0}\right) .
$$

The proof is then concluded by sending $m \rightarrow \infty$ and then $n \rightarrow \infty$ and using (4.21).

4. Since $1 \in \mathcal{U}, \tilde{u}\left(t_{k}, x_{k}, e_{0}\right)$ is bounded from below by

$$
\mathbb{E}\left[g\left(X_{T}^{k}, N_{T}^{k}\right)+\int_{t_{k}}^{T} f\left(X_{s}^{k}, N_{s}^{k}\right) d s-\int_{t_{k}}^{T}\left(\sum_{e \in E} c\left(X_{s-}^{k}, N_{s-}^{k},\left[N_{s-}^{k}+e\right]_{\kappa}\right)\right) d s\right] .
$$

Applying a similar result as (4.24) to a sequence $\left(t_{k}, x_{k}\right)_{k \geq 1}$ in $[0, T) \times \mathbb{R}^{d}$ satisfying $\left(t_{k}, x_{k}\right) \rightarrow\left(T, x_{0}\right)$ and $\tilde{u}\left(t_{k}, x_{k}, e_{0}\right) \rightarrow \tilde{u}_{*}\left(T, x_{0}, e_{0}\right)$ implies that $\tilde{u}_{*}\left(T, x_{0}, e_{0}\right) \geq g\left(x_{0}, e_{0}\right)$. Finally, since $\tilde{u}\left(t_{k}, x_{k}, e_{0}\right) \geq \tilde{u}_{*}\left(t_{k}, x_{k}, e_{0}\right)$ and $\tilde{u}_{*}$ satisfies $\mathcal{G}^{e_{0}} \tilde{u}_{*}\left(t_{k}, x_{k}, e_{0}\right) \geq 0$ for $t_{k}<T$, passing to the limit implies that $\mathcal{G}^{e_{0}} \tilde{u}_{*}\left(\cdot, e_{0}\right) \geq 0$ holds on $[0, T] \times \mathbb{R}^{d}$. 


\subsection{A comparison result}

In this section, we prove a comparison principle for (3.3)-(3.4).

Proposition 4.8 Assume that $\mathbf{H 2}$ holds. Let $U$ (resp. V) be a lower-semicontinuous (resp. upper-semicontinuous) viscosity supersolution (resp. subsolution) of (3.3)-(3.4) such that $V^{+}$and $U^{-}$satisfies the growth condition (3.5). Then, $U \geq V$ on $[0, T] \times \mathbb{R}^{d} \times E$.

Proof. 1. As usual, we shall argue by contradiction. We assume that $\sup _{[0, T] \times \mathbb{R}^{d} \times E}(V-$ $U)>0$. Recalling the definition of $\Lambda$ and $\varrho$ in $\mathbf{H 2}$ of Section 3.3, it follows from the growth condition on $V-U$ that for $\lambda \in(0,1)$ small enough there is some $\left(t_{0}, x_{0}, e_{0}\right) \in$ $[0, T] \times \mathbb{R}^{d} \times E$ such that

$$
\max _{[0, T] \times \mathbb{R}^{d} \times E}(\tilde{V}-\tilde{W})=(\tilde{V}-\tilde{W})\left(t_{0}, x_{0}, e_{0}\right)=: \eta>0
$$

where, for a map $w$ on $[0, T] \times \mathbb{R}^{d} \times E$, we write $\tilde{w}(t, x, e)$ for $e^{\varrho t} w(t, x, e)$, and $\tilde{W}:=$ $(1-\lambda) \tilde{U}+\lambda \tilde{\Lambda}$. Let us define $\tilde{\mathcal{G}}^{e}$ and $\tilde{\mathcal{G}}$ as $\mathcal{G}^{e}$ and $\mathcal{G}$ with $\tilde{c}$ in place of $c$ and observe that $\tilde{U}$ and $\tilde{V}$ are super and subsolutions on $[0, T] \times \mathbb{R}^{d} \times E$ of

$$
\min \{\varrho \varphi-\mathcal{L} \varphi, \tilde{\mathcal{G}} \varphi\}=0
$$

and satisfy the boundary condition

$$
\min \{\varphi-\tilde{g}, \tilde{\mathcal{G}} \varphi\}=0
$$

with $\tilde{g}:=e^{\varrho T} g$.

2. For $(t, x, y, e) \in[0, T] \times \mathbb{R}^{d} \times \mathbb{R}^{d} \times E$ and $n \geq 1$, we set

$$
\begin{aligned}
\Gamma(t, x, y, e) & :=\tilde{V}(t, x, e)-\tilde{W}(t, y, e) \\
\Theta_{n}(t, x, y, e) & :=\Gamma(t, x, y, e)-\left(n|x-y|^{2 \gamma}+\left|x-x_{0}\right|^{2 \gamma+2}+\left|t-t_{0}\right|^{2}+\left|e-e_{0}\right|\right) .
\end{aligned}
$$

By the growth assumption on $V$ and $U$ again, there is $\left(t_{n}, x_{n}, y_{n}, e_{n}\right) \in[0, T] \times \mathbb{R}^{d} \times \mathbb{R}^{d} \times E$ such that

$$
\max _{[0, T] \times \mathbb{R}^{d} \times \mathbb{R}^{d} \times E} \Theta_{n}=\Theta_{n}\left(t_{n}, x_{n}, y_{n}, e_{n}\right)
$$

Since

$$
\Gamma\left(t_{n}, x_{n}, y_{n}, e_{n}\right) \geq \Theta_{n}\left(t_{n}, x_{n}, y_{n}, e_{n}\right) \geq(\tilde{V}-\tilde{W})\left(t_{0}, x_{0}, e_{0}\right)
$$

it follows from the growth assumption on $V$ and $U$, (v) of $\mathbf{H 2 , ~ ( 4 . 2 6 ) ~ a n d ~ t h e ~ u p p e r - ~}$ semicontinuity of $\Gamma$ that, up to a subsequence,

$$
\begin{aligned}
& \left(t_{n}, x_{n}, y_{n}, e_{n}\right) \rightarrow\left(t_{0}, x_{0}, x_{0}, e_{0}\right) \\
& n\left|x_{n}-y_{n}\right|^{2 \gamma}+\left|t_{n}-t_{0}\right|^{2}+\left|e_{n}-e_{0}\right| \rightarrow 0 \\
& \Gamma\left(t_{n}, x_{n}, y_{n}, e_{n}\right) \rightarrow \Gamma\left(t_{0}, x_{0}, x_{0}, e_{0}\right) .
\end{aligned}
$$


3. We first assume that, up to a subsequence,

$$
\tilde{\mathcal{G}}^{e_{n}} \tilde{V}\left(t_{n}, x_{n}, e_{n}\right) \leq 0 \text { for all } n \geq 1 \text {. }
$$

Then, it follows from the supersolution property of $\tilde{U}$ and (iii) of $\mathbf{H 2}$ that, for some $j_{n} \in E \notin\left\{e_{n}\right\}$,

$$
\begin{aligned}
\Gamma\left(t_{n}, x_{n}, y_{n}, e_{n}\right) & \leq \Gamma\left(t_{n}, x_{n}+\beta\left(x_{n}, e_{n}, j_{n}\right), y_{n}+\beta\left(y_{n}, e_{n}, j_{n}\right), j_{n}\right) \\
& +\tilde{c}\left(y_{n}, e_{n}, j_{n}\right)-\tilde{c}\left(x_{n}, e_{n}, j_{n}\right)-\lambda \tilde{q}\left(y_{n}\right) .
\end{aligned}
$$

Observe that $j_{n} \rightarrow j_{0} \in E \backslash\left\{e_{0}\right\}$, up to a subsequence. Using (4.29) and (4.31), we then deduce from the upper-semicontinuity of $\Gamma$, (iii) of $\mathbf{H 2}$ and the continuity of $\tilde{c}$ that

$$
\begin{aligned}
\Gamma\left(t_{0}, x_{0}, x_{0}, e_{0}\right) & <\Gamma\left(t_{0}, x_{0}, x_{0}, e_{0}\right)+\lambda \tilde{q}\left(x_{0}\right) \\
& \leq \Gamma\left(t_{0}, x_{0}+\beta\left(x_{0}, e_{0}, j_{0}\right), x_{0}+\beta\left(x_{0}, e_{0}, j_{0}\right), j_{0}\right)
\end{aligned}
$$

which contradicts the definition of $\left(t_{0}, x_{0}, e_{0}\right)$ in (4.26).

4. We now show that there is a subsequence such that $t_{n}<T$ for all $n \geq 1$. If not, we can assume that $t_{n}=T$ and it follows from the boundary condition (4.28) and the above argument that $\tilde{V}\left(t_{n}, x_{n}, e_{n}\right) \leq \tilde{g}\left(x_{n}, e_{n}\right)$ for all $n \geq 1$, up to a subsequence. Since, by step 1 and (iv) of $\mathbf{H 2}, \tilde{W}\left(t_{n}, y_{n}, e_{n}\right) \geq \tilde{g}\left(y_{n}, e_{n}\right)$, it follows that $\Gamma\left(t_{n}, x_{n}, y_{n}, e_{n}\right) \leq$ $\tilde{g}\left(x_{n}, e_{n}\right)-\tilde{g}\left(y_{n}, e_{n}\right)$. Using (4.29), (4.31) and the continuity of $g$, we then obtain a contradiction to (4.26).

5. In view of the previous arguments, we may assume that

$$
t_{n}<T \quad \text { and } \quad \tilde{\mathcal{G}}^{e_{n}} \tilde{V}\left(t_{n}, x_{n}, e_{n}\right)>0 \text { for all } n \geq 1
$$

Using Ishii's Lemma and following standard arguments, see Theorem 8.3 and the discussion after Theorem 3.2 in [6], we deduce from the viscosity property of $\tilde{U}, \tilde{V}$, (ii) of $\mathbf{H 2}$ and the Lipschitz continuity assumptions on $b, a$ and $f$ that

$$
\varrho \Gamma\left(t_{n}, x_{n}, y_{n}, e_{n}\right) \leq O\left(n\left|x_{n}-y_{n}\right|^{2 \gamma}+\left|x_{n}-y_{n}\right|+\left|x_{n}-x_{0}\right|\right) .
$$

In view of $(4.29),(4.30),(4.31)$, this implies that $\varrho \Gamma\left(t_{0}, x_{0}, x_{0}, e_{0}\right) \leq 0$ which contradicts (4.26).

\section{Some sufficient conditions for $\mathrm{H} 1$ and $\mathrm{H} 2$}

Our general assumptions $\mathbf{H 1}$ and $\mathbf{H 2}$ hold under various different conditions on the coefficients. In this section, we provide some of them.

In the following, $C>0$ is a generic constant which depends only on $T, \kappa$ and $b, a, \beta, f, g$ and $c$. 


\subsection{The growth condition H1}

Observe that, when $c \geq 0$ and $f^{+}+g^{+}$is bounded, then $v, u$ and $\tilde{u}$ are trivially bounded from above so that $\mathbf{H 1}$ is satisfied. We now consider a case where $g$ is upper-bounded by an affine map.

Proposition 5.1 Assume that there exists real constants $C_{1}, C_{2}>0$ and some $\eta \in \mathbb{R}^{d}$ such that

$$
g(x, e) \leq C_{1}+\eta^{\prime} x \quad \text { for all }(x, e) \in \mathbb{R}^{d} \times E \quad, \quad\left[\eta^{\prime} b+f\right]^{+} \leq C_{2} \quad \text { and } \quad \eta^{\prime} \beta-c \leq 0 .
$$

Then, $v^{+}, u^{+}$and $\tilde{u}^{+}$have linear growth.

Proof. The upper bound for $v^{+}$follows immediately from (3.1), (2.4) and the linear growth of $a$. Choosing $\phi$ equal to $a\left(X^{t, x, e}, N^{t, e}\right)^{\prime} \eta$ in the definition of $u$, we see that $u(t, x, e) \leq \eta^{\prime} x+C_{1}+C_{2} T$. The proof is concluded by recalling Remark 3.1.

A similar result can be obtained under weaker conditions on $c$ and $g$ whenever $b, a, f$ are bounded and (3.4) admits a $C^{2}$ solution with bounded derivatives. This follows from the more general result stated in the following proposition.

Proposition 5.2 Assume that there exists a supersolution $w$ to (3.4) satisfying (3.5) such that $w(\cdot, e) \in C^{2}\left(\mathbb{R}^{d}\right)$ for each $e$ and $(\mathcal{L} w)^{+}+\left|D w^{\prime} a\right|$ is uniformly bounded. Then, $v^{+}, u^{+}$and $\tilde{u}^{+}$satisfy (3.5).

Proof. Fix $(t, x, e) \in[0, T] \times \mathbb{R}^{d} \times E$ and $\xi \in \mathcal{S}_{t}(e)$. We write $X$ for $X^{(t, x), \xi}$ and $\tau_{i}$ for $\tau_{i}^{\xi}$. Using Itô's Lemma and the supersolution property of $w$, we obtain that

$$
\begin{aligned}
& w\left(X_{T}, \xi_{T}\right)+\int_{t}^{T} f\left(X_{s}, \xi_{s}\right) d s-\sum_{t<\tau_{i} \leq T} c\left(X_{\tau_{i}-}, \xi_{\tau_{i}-}, \xi_{\tau_{i}}\right) \\
& =w(x, e)+\int_{t}^{T} \mathcal{L}^{\xi_{s}} w\left(X_{s}, \xi_{s}\right) d s+\int_{t}^{T} D w\left(X_{s}, \xi_{s}\right)^{\prime} a\left(X_{s}, \xi_{s}\right) d W_{s} \\
& +\sum_{t<\tau_{i} \leq T}\left[w\left(X_{\tau_{i}-}+\beta\left(X_{\tau_{i}-}, \xi_{\tau_{i}-}, \xi_{\tau_{i}}\right), \xi_{\tau_{i}}\right)-w\left(X_{\tau_{i}-}, \xi_{\tau_{i}-}\right)-c\left(X_{\tau_{i}-}, \xi_{\tau_{i}-}, \xi_{\tau_{i}}\right)\right] \\
& \leq w(x, e)+\int_{t}^{T} \mathcal{L}^{\xi_{s}} w\left(X_{s}, \xi_{s}\right) d s+\int_{t}^{T} D w\left(X_{s}, \xi_{s}\right)^{\prime} a\left(X_{s}, \xi_{s}\right) d W_{s} .
\end{aligned}
$$

Since $\mathcal{L} w^{+}$and $\left|D w^{\prime} a\right|$ are uniformly bounded by some $C>0$ and $g \leq w$ we deduce that 1. if $\xi=N^{t, e}$, we can take $\phi:=a\left(X^{t, x, e}, N^{t, e}\right)^{\prime} D w\left(X^{t, x, e}, N^{t, e}\right)$ in the definition of $u$ and obtain the upper bound $w(x, e)+T C$. In view of Remark 3.1 this also provides an upper bound for $\tilde{u}$.

2. the expectation in the definition of $v$ is bounded by $w(x, e)+T C$, uniformly in $\xi \in \mathcal{S}_{t}(e)$. 
We conclude this section with a last condition which pertains for unbounded coefficients but imposes a restriction on the support of $\beta$ and the sign of $c$. It only provides an upper bound for $v$ and $\tilde{u}$.

Proposition 5.3 Assume that

$$
c \geq 0 \quad \text { and } \quad \beta=0 \quad \text { on }\left(\left\{x \in \mathbb{R}^{d}:|x| \geq K\right\} \times E^{2}\right),
$$

for some $K>0$. Then $v^{+}$and $\tilde{u}^{+}$satisfy the growth condition (3.5) with $\gamma:=\bar{p}$.

Proof. We only treate the upper bound of $v$. Similar arguments lead to an upper bound for the expectation in (3.2), uniformly in $\nu \in \mathcal{U}$. For ease of notations, we write $X$ for $X^{(t, x), \xi}$ and $\tau_{i}$ for $\tau_{i}^{\xi}$. It follows from the assumption $c \geq 0$ and (2.6) that

$\mathbb{E}\left[g\left(X_{T}, \xi_{T}\right)+\int_{t}^{T} f\left(X_{s}, \xi_{s}\right) d s-\sum_{t<\tau_{i} \leq T} c\left(X_{\tau_{i}-}, \xi_{\tau_{i}-}, \xi_{\tau_{i}}\right)\right] \leq C\left(1+\sup _{t \leq s \leq T} \mathbb{E}\left[\left|X_{s}\right|^{\bar{p}}\right]\right)$.

Thus, it suffices to show that

$$
\sup _{t \leq s \leq T} \mathbb{E}\left[\left|X_{s}\right|^{\bar{p}}\right] \leq C\left(1+|x|^{\bar{p}}\right)
$$

Since $\beta$ is uniformly Lipschitz, it follows from its support condition that is it bounded by some constant $K^{\prime}>0$. Fix $K^{\prime \prime}:=K+K^{\prime}+|x|$. Let us introduce the sequence of stopping times $\left(\vartheta_{i}\right)_{i \geq 1}$ by $\vartheta_{1}=\inf \left\{s \geq t:\left|X_{s}\right| \geq 2 K^{\prime \prime}\right\}$ and for $i \geq 1$

$$
\vartheta_{2 i}:=\inf \left\{s \geq \vartheta_{2 i-1}:\left|X_{s}\right| \leq K "\right\} \quad, \quad \vartheta_{2 i+1}:=\inf \left\{s \geq \vartheta_{2 i}:\left|X_{s}\right| \geq 2 K "\right\} .
$$

It follows from (5.1) that $\left|X_{\vartheta_{2 i+1}}\right|=2 K$ ". Fix $s \in[t, T]$ and set $A_{s}^{i}:=\left\{\vartheta_{2 i-1} \leq s<\vartheta_{2 i}\right\}$, $A_{s}:=\cup_{i \geq 1} A_{s}^{i}$ and $B_{u}^{s, i}:=\left\{\vartheta_{2 i-1} \leq u \leq s<\vartheta_{2 i}\right\}$. Then

$$
X_{s} \mathbf{1}_{A_{s}}=\sum_{i \geq 1}\left(X_{\vartheta_{2 i-1}} \mathbf{1}_{A_{s}^{i}}+\int_{t}^{s} \mathbf{1}_{B_{u}^{s, i}} b\left(X_{u}, \xi_{u}\right) d u+\int_{t}^{s} \mathbf{1}_{B_{u}^{s, i}} a\left(X_{u}, \xi_{u}\right) d W_{u}\right),
$$

and it follows from the Lipschitz continuity of $b$ and $a$ that

$$
\mathbb{E}\left[\left|X_{s} \mathbf{1}_{A_{s}}\right|^{2 \bar{p}}\right] \leq C\left(1+\left(K^{\prime \prime}\right)^{2 \bar{p}}+\int_{t}^{s} \mathbb{E}\left[\sum_{i \geq 1} \mathbf{1}_{B_{u}^{s, i}}\left|X_{u}\right|^{2 \bar{p}}\right] d u\right) .
$$

Since $B_{u}^{s, i} \subset \tilde{B}_{u}:=\left\{\left|X_{u}\right| \geq K^{\prime \prime}\right\}$ and $\tilde{B}_{s} \subset A_{s} \cup\left\{\left|X_{s}\right| \leq 2 K^{\prime \prime}\right\}$, we get

$$
\mathbb{E}\left[\left|X_{s} \mathbf{1}_{\tilde{B}_{s}}\right|^{2 \bar{p}}\right] \leq C\left(1+\left(K^{\prime \prime}\right)^{2 \bar{p}}+\int_{t}^{s} \mathbb{E}\left[\mathbf{1}_{\tilde{B}_{u}}\left|X_{u}\right|^{2 \bar{p}}\right] d u\right) .
$$

It then follows from Gronwall's Lemma that

$$
\mathbb{E}\left[\left|X_{s}\right|^{2 \bar{p}}\right] \leq\left(K^{\prime \prime}\right)^{2 \bar{p}}+\mathbb{E}\left[\left|X_{s} \mathbf{1}_{\tilde{B}_{s}}\right|^{2 \bar{p}}\right] \leq C\left(1+\left(K^{\prime \prime}\right)^{2 \bar{p}}\right) .
$$

Since $K^{\prime \prime}=K+K^{\prime}+|x|$, this leads to the required result. 


\subsection{The strict supersolution condition $\mathrm{H} 2$}

We now provide a general condition under which $\mathbf{H 2}$ holds.

Proposition 5.4 Fix some integer $\gamma \geq \bar{p}$. Assume that there is a sequence of real numbers $\left(d_{i}\right)_{i \in E}$ and some $\alpha>0$ such that

$$
\begin{aligned}
-\alpha & <|x+\beta(x, i, j)|^{2 \gamma}-|x|^{2 \gamma} \quad \text { for all }(x, i, j) \in \mathbb{R}^{d} \times E^{2} \\
\eta & :=\min _{i, j \in E} \inf _{x \in \mathbb{R}^{d}} \frac{d_{i}-d_{j}+c(x, i, j)}{|x+\beta(x, i, j)|^{2 \gamma}-|x|^{2 \gamma}+\alpha}>0 .
\end{aligned}
$$

Then, assumption $\mathbf{H 2}$ holds for $\gamma$.

Proof. We set $\Lambda(x, e):=\left(d+\eta|x|^{2 \gamma}+d_{e}\right)$ for some $d>0$ large enough so that $\Lambda \geq g^{+}$, recall (2.6). A straightforward computation shows that (iii) of $\mathbf{H 2}$ is satisfied with $q \equiv \alpha \eta$. Clearly, (i) and (v) hold too. Finally, it follows from the linear growth assumption on $b$ and $a$ that (ii) holds for a sufficiently large parameter $\varrho$.

Remark 5.1 (i) If $c \geq \varepsilon$ for some $\varepsilon>0$ and $\beta$ satisfies the support condition of Proposition 5.3, then the conditions of Proposition 5.4 trivially hold with $d_{i}=0$ for all $i \in E$ and $\alpha$ large enough.

(ii) In [13], the authors consider the case where $\beta \equiv 0$ and $c$ satisfies a strict triangular condition

$$
c(x, i, j)+c(x, j, k)>c(x, i, k) \quad \text { for all } x \in \mathbb{R}^{d}, i, j, k \in E .
$$

When $c$ does not depend on $x$, they show that the sequence $\left(d_{i}\right)_{i \in E}$ defined by

$$
d_{i}=\min _{j \in E \backslash\{i\}} c(x, i, j)
$$

satisfies $d_{i}-d_{j}+c(x, i, j)>0$, see the first step of their Theorem 4.3 in Appendix. It follows that if $c$ is independent of $x$, satisfies (5.2) and $\beta$ satisfies the support condition of Proposition 5.3 or more generally the first condition of Proposition 5.4, then the second condition of this proposition holds too with $\left(d_{i}\right)_{i \in E}$ defined as in (5.3) and $\alpha$ large enough.

\section{References}

[1] Bensoussan A. and J.-L. Lions (1984), Impulse control and quasi-variational inequalities, Gauthier-Villars.

[2] Bouchard B. (2002), Stochastic Targets with Mixed diffusion processes, Stochastic Processes and their Applications, 101, 273-302. 
[3] Bouchard B. and R. Elie (2005), Discrete time approximation of decoupled ForwardBackward SDE with jumps, preprint LPMA, University Paris 6.

[4] Brekke K. and B. Oksendal (1994), Optimal switching in an economic activity under uncertainty, SIAM J. Cont. Optim., 32, 1021-1036.

[5] Brémaud P. (1981), Point Processes and Queues - Martingale Dynamics, SpringerVerlag, New-York.

[6] Crandall M.G., H. Ishii and P.-L. Lions (1992), User's guide to viscosity solutions of second order Partial Differential Equations, Amer. Math. Soc., 27, 1-67.

[7] Delbaen F. et W. Schachermayer (1994). A general version of the fundamental theorem of asset pricing, Math. Annalen, 300, 463-520.

[8] Dixit A. and R. Pindick (1994), Investment under uncertainty, Princeton University Press.

[9] Duckworth K. and M. Zervos (2001, A model for investment decisions with switching costs, Annals of Applied Probability, 11, 239-250.

[10] Guo X. (2001), An explicit solution to an optimal stopping problem with regime switching, Journal of Applied Probability, 38, 464-481.

[11] El Karoui N. and M.-C. Quenez (1995), Dynamic Programming and Pricing of Contingent Claims in an Incomplete Market, SIAM J. on Control and Optimization, $33(1), 22-66$.

[12] Kramkov D. (1996), Optional decomposition of supermartingales and hedging contingent claims in incomplete security markets, Probability Theory and Related Fields, $105(4), 459-479$.

[13] Ly Vath V. and H. Pham (2006), Explicit solution to an optimal switching problem in the two-regime case, preprint LPMA, University Paris 7.

[14] Pardoux E., F. Pradeilles and Z. Rao (1997), Probabilistic interpretation for a system of semilinear parabolic partial differential equations, Ann. Inst. H. Poincare, 33 (4), 467-490.

[15] Protter P. (1990), Stochastic Integration and Differential Equations, SpringerVerlag.

[16] Soner H. M. and N. Touzi (2002), Stochastic target problems, dynamic programming and viscosity solutions, SIAM Journal on Control and Optimization, 41, 404-424. 
[17] Soner H. M. and N. Touzi (2002), Dynamic programming for stochastic target problems and geometric flows, Journal of the European Mathematical Society, 4, 201-236.

[18] Sow A. B. and E. Pardoux (2004), Probabilistic interpretation of a system of quasilinear parabolic PDEs, Stochastics and Stochastics Reports, 76 (5), 429-477.

[19] Tang S. and J. Yong (1993), Finite horizon stochastic optimal switching and impulse controls with a viscosity solution approach, Stoch. and Stoch. Reports, 45, 145-176. 